\title{
PATCHY VECTOR FIELDS AND ASYMPTOTIC STABILIZATION
}

\author{
FAbio AnCona ${ }^{1}$ And Alberto Bressan ${ }^{2}$
}

\begin{abstract}
This paper is concerned with the structure of asymptotically stabilizing feedbacks for a nonlinear control system on $\mathbb{R}^{n}$. We first introduce a family of discontinuous, piecewise smooth vector fields and derive a number of properties enjoyed by solutions of the corresponding O.D.E's. We then define a class of "patchy feedbacks" which are obtained by patching together a locally finite family of smooth controls. Our main result shows that, if a system is asymptotically controllable at the origin, then it can be stabilized by a piecewise constant patchy feedback control.

Résumé. Dans cet article, on considère la structure de lois de feedback qui stabilisent asymptotiquement un système de contrôle non linéaire. Nous étudions une famille de champs de vecteurs discontinus, réguliers par morceaux, et démontrons de nombreuses propriétés satisfaites par les équations différentielles ordinaires correspondantes. En outre, nous définissons une classe de "feedbacks rapiécés" qui sont obtenus par la superposition d'une famille localement finie de contrôles réguliers. Notre résultat principal montre que, si le système est asymptotiquement contrôlable à l'origine, alors il peut être stabilisé par un "feedback rapiécé", constant par morceaux.
\end{abstract}

AMS Subject Classification. 34A, 34D, 49E, 93D.

Received December 22, 1998. Revised May 15, 1999.

\section{INTRODUCTION}

Consider the control system on $\mathbb{R}^{n}$

$$
\dot{x}=f(x, u) \quad u(t) \in K,
$$

assuming that control set $K \subset \mathbb{R}^{m}$ is compact and that the map $f: \mathbb{R}^{n} \times \mathbb{R}^{m} \mapsto \mathbb{R}^{n}$ is smooth. We are concerned with the classical problem of finding a feedback control $u=U(x) \in K$ such that all trajectories of the corresponding O.D.E.

$$
\dot{x}=f(x, U(x))
$$

tend to the origin as $t \rightarrow \infty$.

Assume that, for every initial data $\bar{x} \in \mathbb{R}^{n}$, there exists an open loop control $u=u(t, \bar{x})$ such that the solution of

$$
\dot{x}=f(x, u(t, \bar{x})), \quad x(0)=\bar{x}
$$

Keywords and phrases: Asymptotic controllability, feedback asymptotic stabilization, discontinuous feedback, discontinuous vector fields.

${ }^{1}$ Dipartimento di Matematica and CIRAM, Università di Bologna, piazza Porta S. Donato 5, Bologna 40127, Italy;

e-mail: ancona@ciram3.ing.unibo.it

2 S.I.S.S.A., via Beirut 4, Trieste 34014, Italy; e-mail: bressan@sissa.it 
asymptotically tends to the origin. If the system is nonlinear, it is well known that there may not be any continuous feedback which asymptotically steers to the origin every point $\bar{x} \in \mathbb{R}^{n}$. Indeed, the possible nonexistence of such feedbacks was first brought to light in [28] for a two-dimensional system $\left(n=2, K=\mathbb{R}^{2}\right)$, and in [23] for one-dimensional systems $(n=1, K=R)$. General results, regarding multidimensional systems, were presented in $[3,9,28]$, where certain topological obstructions that can prevent the existence of continuous stabilizing feedback were discovered.

It is thus natural to look for a stabilizing control within a class of discontinuous functions. The pioneer work in this direction was [28], where it was shown that any controllable analytic system can be asymptotically stabilized by means of piecewise analytic feedback laws, if one prescribes suitable "exit rules" (that cannot be expressed in terms of a true feedback) on the singular set of the feedback controls. However, allowing nonregular feedbacks, immediately leads to a major theoretical difficulty. Namely, when the function $U$ is discontinuous, the differential equation (1.2) may not have any solution. To overcome this problem, two approaches are possible.

1. On one hand, one can choose to work with arbitrary feedback controls $u=U(x)$. In this case, there is no guarantee that (1.2) will have any solution in the usual Carathéodory sense. Therefore, one must introduce some new definition of "generalized solution" of an O.D.E. with arbitrary measurable right hand side and show that, with this definition, stabilizing trajectories always exist.

2. On the other hand, one can single out a family of discontinuous feedbacks whose singularities are sufficiently tame, so that the corresponding differential equation (1.2) always admits Carathéodory solutions. One then has to prove that a stabilizing feedback exists within this particular class of functions.

The first approach was taken in [4], considering a family of approximate solutions obtained by "sampling" the feedback control at discrete times, then applying a constant control between two consecutive sampling times and thus constructing an approximate solution of the corresponding O.D.E. The existence of a stabilizing feedback in this generalized sense is the main result in [4], where the regularity of the feedback was not a matter of concern. It is remarkable that the definitions of Filippov or Krasovskii generalized solutions, frequently encountered in the literature, cannot be used here. Indeed, for a given initial data, these generalized solutions form a closed and connected set. The same topological obstructions to the existence of a continuous feedback are thus encountered in this case $[10,22]$.

In the present paper, we follow the second approach. First we introduce a particular class of discontinuous vector fields. These are called "patchy" because they are obtained by patching together smooth vector fields defined on a locally finite family of positively invariant regions. The analysis of differential equations with patchy right hand side reveals many nice properties. If $g$ is a patchy vector field, for any initial data $\bar{x}$ the Cauchy problem

$$
\dot{x}=g(x), \quad x(0)=\bar{x}
$$

has at least one forward solution and at most one backward solution, in the Carathéodory sense. These solutions are not only absolutely continuous, but in fact piecewise $\mathcal{C}^{1}$. Moreover, the set of all solutions is closed in the topology of uniform convergence, but possibly not connected. For patchy vector fields, any generalized solution obtained as a limit of the approximate solutions considered in [4] is a Carathéodory solution as well.

We then define a "patchy feedback" as a piecewise constant feedback control $U$ such that $g(x) \doteq f(x, U(x))$ determines a patchy vector field. Our main theorem shows that, if the system (1.1) is asymptotically controllable to the origin, then it can be stabilized by a patchy feedback. This provides an alternative proof of the result in [4] which is conceptually simpler. Indeed, the construction of the stabilizing feedback here is completely independent from the existence of a control-Lyapunov functional for (1.1). On the other hand, it achieves a feedback control with better regularity properties.

\section{BASIC NOTATIONS AND DEFINITIONS}

In the following, by $B(x, r)$ we denote the closed ball centered at $x$ with radius $r$. The closure, the interior and the boundary of a set $\Omega$ are written as $\bar{\Omega}, \stackrel{\circ}{\Omega}$ and $\partial \Omega$, respectively. 
Definition 2.1. Let $\Omega \subset \mathbb{R}^{n}$ be an open domain with smooth boundary $\partial \Omega$. We say that a smooth vector field $g$ defined on a neighborhood of $\bar{\Omega}$ is an inward-pointing vector field on $\Omega$ if at every boundary point $x \in \partial \Omega$ the inner product of $g$ with the outer normal $\mathbf{n}$ satisfies

$$
\langle g(x), \mathbf{n}(x)\rangle<0
$$

The pair $(\Omega, g)$ will be called a patch.

A vector field on $\Omega \subset \mathbb{R}^{n}$ defined as the superposition of inward-pointing vector fields will be called a patchy vector field. More precisely:

Definition 2.2. We say that $g: \Omega \mapsto \mathbb{R}^{n}$ is a patchy vector field if there exists a family of patches $\left\{\left(\Omega_{\alpha}, g_{\alpha}\right)\right.$ : $\alpha \in \mathcal{A}\}$ such that

- $\mathcal{A}$ is a totally ordered index set,

- the open sets $\Omega_{\alpha}$ form a locally finite covering of $\Omega$,

- the vector field $g$ can be written in the form

$$
g(x)=g_{\alpha}(x) \quad \text { if } \quad x \in \Omega_{\alpha} \backslash \bigcup_{\beta>\alpha} \Omega_{\beta} .
$$

We shall occasionally adopt the longer notation $\left(\Omega, g,\left(\Omega_{\alpha}, g_{\alpha}\right)_{\alpha \in \mathcal{A}}\right)$ to indicate a patchy vector field, specifying both the domain and the single patches. By defining

$$
\alpha^{*}(x) \doteq \max \left\{\alpha \in \mathcal{A}: x \in \Omega_{\alpha}\right\}
$$

we thus have the equivalent definition

$$
g(x)=g_{\alpha^{*}(x)}(x) \quad \forall x \in \Omega
$$

Remark 2.1. Of course, the patches $\left(\Omega_{\alpha}, g_{\alpha}\right)$ are not uniquely determined by the patchy vector field $g$. Indeed, whenever $\alpha<\beta$, by (2.2) the values of $g_{\alpha}$ on the set $\Omega_{\alpha} \cap \Omega_{\beta}$ are irrelevant. In the construction of patchy vector fields, the following observation is often useful. Assume that the open sets $\Omega_{\alpha}$ form a locally finite covering of $\Omega$ and that, for each $\alpha \in \mathcal{A}$, the vector field $g_{\alpha}$ satisfies (2.1) at every point $x \in \partial \Omega_{\alpha} \backslash \bigcup_{\beta>\alpha} \Omega_{\beta}$. Then $g$ is again a patchy vector field. To see this, it suffices to construct vector fields $\tilde{g}_{\alpha}$ which satisfy the inward pointing property (2.1) at every point $x \in \partial \Omega_{\alpha}$ and such that $\tilde{g}_{\alpha}=g_{\alpha}$ on $\Omega_{\alpha} \backslash \bigcup_{\beta>\alpha} \Omega_{\beta}$. To accomplish this, for each $\alpha$ we first consider a smooth vector field $v_{\alpha}$ such that $v_{\alpha}(x)=-\mathbf{n}(x)$ on $\partial \Omega_{\alpha}$. Then we construct a smooth scalar function $\varphi_{\alpha}: \Omega \mapsto[0,1]$ such that

$$
\varphi_{\alpha}(x)=\left\{\begin{array}{lll}
1 & \text { if } & x \in \Omega_{\alpha} \backslash \bigcup_{\beta>\alpha} \Omega_{\beta}, \\
0 & \text { if } \quad x \in \partial \Omega_{\alpha},\langle g(x), \mathbf{n}(x)\rangle \geq 0 .
\end{array}\right.
$$

Finally, for each $\alpha \in \mathcal{A}$ we define the interpolation

$$
\tilde{g}_{\alpha}(x) \doteq \varphi_{\alpha}(x) g_{\alpha}(x)+\left(1-\varphi_{\alpha}(x)\right) v_{\alpha}(x) .
$$

The vector fields $\tilde{g}_{\alpha}$ thus defined satisfy our requirements.

We recall that a Carathéodory solution to

$$
\dot{x}=g(x)
$$

on an interval $I \subset \mathbb{R}$ by definition is an absolutely continuous function $x: I \mapsto \Omega$ which satisfies (2.4) almost everywhere on $I$. This holds if and only if, for every $t_{0} \in I$, one has

$$
x(t)=x\left(t_{0}\right)+\int_{t_{0}}^{t} g(x(s)) d s \quad \forall t \in I .
$$


For any fixed $x_{0} \in \Omega$, we shall denote with $S_{C}\left(x_{0}\right)$ the set of all forward Carathéodory solution $x(\cdot)$ to $(2.4)$ with initial condition $x(0)=x_{0}$ defined on some interval $[0, T)$. Moreover we call $S_{C}^{\max }\left(x_{0}\right)$ the set of all maximal forward Carathéodory solution to (2.4) with initial condition $x(0)=x_{0}$, i.e. the set of all absolutely continuous function $\gamma(\cdot) \in S_{C}\left(x_{0}\right)$ such that one of the following two cases holds:

i) the map $\gamma(\cdot)$ is defined on $[0, \infty)$. In this case we set $\tau^{\max }(\gamma) \doteq \infty$.

ii) The map $\gamma(\cdot)$ is defined on $[0, T)$ for some $T>0$ such that

$$
\limsup _{t \rightarrow T^{-}}\left(|\gamma(t)|+\frac{1}{d(\gamma(t), \partial \Omega)}\right)=\infty .
$$

In this case we set $\tau^{\max }(\gamma) \doteq T$.

As in $[4,19]$, we shall also consider a perturbed system associated to $(2.4)$

$$
\dot{x}=g(x+\eta(t))+\zeta(t)
$$

where $t \rightarrow \eta(t), t \rightarrow \zeta(t)$ are integrable functions representing, respectively, a measurement error (in state estimation) and an external disturbance. In connection with (2.5) we now introduce a definition of "Euler polygonal solution" which takes into account initial measurement errors and external disturbances. Let

$$
\pi=\left\{a=\tilde{t}_{0}<\tilde{t}_{1}<\cdots<\tilde{t}_{N_{\pi}}=b\right\}
$$

be a partition of the interval $[a, b]$. Denote

$$
\delta_{\pi} \doteq \sup _{0 \leq i<N_{\pi}}\left(\tilde{t}_{i+1}-\tilde{t}_{i}\right)
$$

its (maximal) mesh size and consider an $N_{\pi}$-tuple of elements in $\mathbb{R}^{n}$

$$
\left\{c_{i}: 0 \leq i<N_{\pi}\right\},
$$

which represents a discrete external disturbance of the dynamic described by (2.4).

Definition 2.3. In the above setting, given any $e \in \mathbb{R}^{n}$, a function $x_{\pi}:[a, b] \mapsto \Omega$ recursively defined by

$$
\begin{array}{lll}
x_{\pi}(t)=x_{\pi}(a)+\left(g\left(x_{\pi}(a)+e\right)+c_{0}\right)(t-a) & t \in\left[a, \tilde{t}_{1}\right], & \\
x_{\pi}(t)=x_{\pi}\left(\tilde{t}_{i}\right)+\left(g\left(x_{\pi}\left(\tilde{t}_{i}\right)\right)+c_{i}\right)\left(t-\tilde{t}_{i}\right) & t \in\left[\tilde{t}_{i}, \tilde{t}_{i+1}\right], & 0<i<N_{\pi},
\end{array}
$$

will be called a forward Euler $\pi$-solution of (2.4) perturbed by the external disturbance

$$
\zeta_{\pi} \doteq \sup _{0 \leq i<N_{\pi}}\left|c_{i}\right|,
$$

and the initial measurement error $\eta_{\pi} \doteq|e|$.

In this framework it will be also useful to introduce the following definition of "perturbed" patchy vector field. 
Definition 2.4. Let $\left(\Omega, g,\left(\Omega_{\alpha}, g_{\alpha}\right)_{\alpha \in \mathcal{A}}\right)$ be a smooth patchy vector field, and $\chi: \Omega \mapsto R^{+}$a continuous function. Assume that, for any single patch $\left(\Omega_{\alpha}, g_{\alpha}\right), \alpha \in \mathcal{A}$, there holds

$$
\left\langle g_{\alpha}(x)+v, \mathbf{n}(x)\right\rangle<0 \quad \text { whenever } x \in \partial \Omega_{\alpha},|v| \leq \chi(x) .
$$

Then, we say that the multivalued map $G: \Omega \mapsto 2^{\mathbb{R}^{n}}$ defined by

$$
G(x) \doteq g(x)+B(0, \chi(x)) \quad x \in \Omega,
$$

is an admissible multivalued perturbation of $g$.

Remark 2.2. Notice that, in view of Remark 2.1, in the definition of an admissible multivalued perturbation $G$ associated to a patchy vector field $\left(\Omega, g,\left(\Omega_{\alpha}, g_{\alpha}\right)_{\alpha \in \mathcal{A}}\right)$, it is sufficient to require that each vector field $g_{\alpha}$ satisfies (2.8) for every $x \in \partial \Omega_{\alpha} \backslash \bigcup_{\beta>\alpha} \Omega_{\beta},|v| \leq \chi(x)$.

In connection with the control system (1.1), we first recall the definition of global null-asymptotic controllability, then we introduce a class of piecewise constant feedback control $U=U(x)$ such that $g(x)=f(x, U(x))$ is a patchy vector field which asymptotically stabilizes the system (1.2) at the origin. We consider as (open-loop) admissible controls all the measurable functions $u:[0, \infty) \rightarrow \mathbb{R}^{m}$ such that $u(t) \in K$ for a.e. $t \in[0, \infty)$. For any fixed $x_{0} \in \mathbb{R}^{n}$ and any admissible control $u_{0}$, we denote $x\left(\cdot ; x_{0}, u_{0}\right)$ the forward Carathéodory solution to (1.1) (i.e. the absolutely continuous function that satisfies (1.1) for a.e. $t>0$ ) with initial condition $x(0)=x_{0}$ and control $u=u_{0}$, defined on some maximal interval $\left[0, \tau^{\max }\left(x_{0}, u_{0}\right)\right)$.

Definition 2.5. The system (1.1) is said to be globally asymptotically controllable (to the origin) if the following holds.

1. Attractiveness: for each $x_{0} \in \mathbb{R}^{n}$ there exists some admissible control $u_{0} \doteq u_{x_{0}}$ such that the trajectory $t \rightarrow x(t) \doteq x\left(t ; x_{0}, u_{0}\right)$ is defined for all $t \geq 0$, i.e. $\tau^{\max }\left(x_{0}, u_{0}\right)=\infty$, and such that $x(t) \rightarrow 0$ as $t \rightarrow \infty$.

2. Lyapunov stability: for each $\varepsilon>0$ there exists $\delta>0$ such that for each $x_{0} \in \mathbb{R}^{n}$ with $\left|x_{0}\right|<\delta$ there is an admissible control $u_{0}$ as in 1 . such that $|x(t)|<\varepsilon$ for all $t \geq 0$.

Definition 2.6. Let $\left(\Omega, g,\left(\Omega_{\alpha}, g_{\alpha}\right)_{\alpha \in \mathcal{A}}\right)$ be a patchy vector field. Assume that there exist control values $k_{\alpha} \in K$ such that, for each $\alpha \in \mathcal{A}$

$$
g_{\alpha}(x) \doteq f\left(x, k_{\alpha}\right) \quad \forall x \in \Omega_{\alpha} \backslash \bigcup_{\beta>\alpha} \Omega_{\beta} .
$$

Then the piecewise constant map

$$
U(x) \doteq k_{\alpha} \quad \text { if } \quad x \in \Omega_{\alpha} \backslash \bigcup_{\beta>\alpha} \Omega_{\beta} .
$$

is called a patchy feedback control on $\Omega$.

Remark 2.3. From Definitions 2.2 and 2.5, in view of Remark 2.1, it is clear that the field

$$
g(x)=f(x, U(x))
$$


defined in connection with a given patchy feedback $\left(\Omega, U,\left(\Omega_{\alpha}, k_{\alpha}\right)_{\alpha \in \mathcal{A}}\right)$ is precisely the patchy vector field $\left(\Omega, g,\left(\Omega_{\alpha}, g_{\alpha}\right)_{\alpha \in \mathcal{A}}\right)$ associated with a family of fields $\left\{g_{\alpha}: \alpha \in \mathcal{A}\right\}$ satisfying (2.10). Moreover, recalling the notation (2.3) we have

$$
U(x)=k_{\alpha^{*}(x)} \quad \forall x \in \Omega .
$$

Definition 2.7. A patchy feedback control $U: \mathbb{R}^{n} \backslash\{0\} \mapsto K$ is said to asymptotically stabilize the closed-loop system (1.2) with respect to the origin if the following holds.

1. Uniform attractiveness: for each $x_{0} \in \mathbb{R}^{n} \backslash\{0\}$ and for every Carathéodory trajectory $\gamma$ of (1.2) starting from $x_{0}$ one has

$$
\lim _{t \rightarrow \tau^{\max }(\gamma)} \gamma(t)=0
$$

2. Lyapunov stability: for each $\varepsilon>0$ there exists $\delta>0$ such that, for each $x_{0} \in \mathbb{R}^{n} \backslash\{0\}$ with $\left|x_{0}\right|<\delta$ and for any Carathéodory trajectory $\gamma$ of (1.2) starting from $x_{0}$, one has

$$
|\gamma(t)|<\varepsilon \quad \forall 0 \leq t<\tau^{\max }(\gamma) .
$$

Next, we shall investigate robustness properties of a stabilizing patchy feedback $U: \mathbb{R}^{n} \backslash\{0\} \mapsto K$ with respect to small external perturbations of the system dynamics (1.2). This problem is best recasted in terms of differential inclusions as follows. Let $\chi: \mathbb{R}^{n} \mapsto \mathbb{R}^{+}$be a continuous map and consider the differential inclusion

$$
\dot{x} \in f(x, U(x))+B(0, \chi(x))
$$

associated to the system (1.2). By a Caratéodory trajectory of (2.15) we mean an absolutely continuous function $x: I \mapsto \mathbb{R}^{n} \backslash\{0\}$, defined on some interval $I \subset \mathbb{R}$, which satisfies (2.15) for a.e. $t \in I$. This is equivalent to say that there exists a measurable map $\zeta: I \mapsto \mathbb{R}^{n}$ such that

$$
|\zeta(t)| \leq \chi(x(t)) \quad \text { a.e. } t \in I
$$

and for which $x(\cdot)$ is Caratéodory solution of the perturbed system

$$
\dot{x}=f(x, U(x))+\zeta(t) .
$$

Definition 2.8. Let $U: \mathbb{R}^{n} \backslash\{0\} \mapsto K$ be a patchy feedback control that asymptotically stabilize the closedloop system (1.2) with rispect to the origin. We say that $U$ is robust with respect to external disturbances if there exists some strictly positive continuous function $\chi: \mathbb{R}^{n} \backslash\{0\} \mapsto \mathbb{R}^{+}$such that $\mathbf{1}$. and 2 . in Definition 2.7 hold also for the trajectories $\gamma$ of (2.15).

\section{TRAJECTORIES OF PATCHY VECTOR FIELDS}

The next proposition collects the main properties of trajectories of patchy vector fields.

Proposition 3.1. Let $\left(\Omega, g,\left(\Omega_{\alpha}, g_{\alpha}\right)_{\alpha \in \mathcal{A}}\right)$ be a patchy vector field on an open domain $\Omega$. Then the following holds.

(i) If $t \rightarrow x(t)$ is a Carathéodory solution to (2.4) on an interval $J$, then $t \rightarrow \dot{x}(t)$ is piecewise smooth and has a finite set of jumps on any compact subset $J^{\prime} \subset J$. The function $t \mapsto \alpha^{*}(x(t))$ defined at (2.3) is non-decreasing and left continuous. Moreover there holds

$$
\dot{x}(t-)=g(x(t)) \quad \forall t \in \stackrel{\circ}{J} .
$$


(ii) For each $x_{0} \in \Omega, t_{0} \in \mathbb{R}$, the Cauchy problem (2.4) with initial condition $x\left(t_{0}\right)=x_{0}$ has at least one local forward Carathéodory solution and at most one backward Carathéodory solution.

(iii) The set of Carathéodory solutions of (2.4) is closed. More precisely, if $x_{n}:\left[a_{n}, b_{n}\right] \mapsto \Omega$ is a sequence of solutions and, as $n \rightarrow \infty$ one has

$$
\left.a_{n} \rightarrow a, \quad b_{n} \rightarrow b, \quad x_{n}(t) \rightarrow \hat{x}(t) \quad \forall t \in\right] a, b[,
$$

then $\hat{x}(\cdot)$ is itself a Carathéodory solution of (2.4).

(iv) A function $t \rightarrow x(t)$ is a Carathéodory solution to (2.4) if and only if it is the uniform limit on compact sets, as mesh size tends to zero, of a sequence $\left(x_{\pi_{n}}\right)_{n}$ of forward Euler $\pi$-solution to (2.4) perturbed by initial measurement errors $\left(\eta_{\pi_{n}}\right)_{n}$ and external disturbances $\left(\zeta_{\pi_{n}}\right)_{n}$ with

$$
\lim _{n \rightarrow \infty} \eta_{\pi_{n}}=0, \quad \quad \lim _{n \rightarrow \infty} \zeta_{\pi_{n}}=0 .
$$

Proof. To prove (i), it is sufficient to show that $t \rightarrow \dot{x}(t)$ has a locally finite set of jumps and satisfies (3.1) since then one can derive the conclusion by a standard compactness argument. Thus, given any $\tau \in J$, recalling that $\left\{\Omega_{\alpha}: \alpha \in \mathcal{A}\right\}$ is a locally finite covering of $\Omega$ we can choose some interval $(\tau-\delta, \tau+\delta] \subset J$, which intersects only finitely many elements of $\left\{x^{-1}\left(\Omega_{\alpha}\right): \alpha \in \mathcal{A}\right\} \quad$ (in the case $\tau=\inf J$, choose an interval $(\tau, \tau+\delta]$ with such a property). Then, set

$$
\left\{\alpha_{1}, \ldots, \alpha_{I}\right\} \doteq\left\{\alpha \in \mathcal{A}:(\tau-\delta, \tau+\delta] \cap x^{-1}\left(\Omega_{\alpha}\right) \neq \emptyset\right\}
$$

We will show that $t \rightarrow \dot{x}(t)$ has a finite number of jumps in $(\tau, \tau+\delta]$. To this end, observe that each $E_{\alpha_{i}} \doteq x^{-1}\left(\Omega_{\alpha}\right), 1 \leq i \leq I$, being an open subset of $\mathbb{R}$, is a countable disjoint union of open intervals, say $J_{\ell}^{\alpha_{i}}, \ell \in \mathbb{N}$. Moreover, from the definition of $g$ it follows that, for any pair of intervals $J_{\ell}^{\alpha_{i}}, J_{h}^{\alpha_{j}}$ with non empty intersection and such that $\alpha_{i} \neq \alpha_{j}$, there holds

$$
\alpha^{*}(x(t)) \leq \alpha_{j} \quad \forall t \in J_{\ell}^{\alpha_{i}} \quad \Longrightarrow \quad \sup J_{\ell}^{\alpha_{i}} \leq \sup J_{h}^{\alpha_{j}}
$$

since otherwise one would have $x\left(J_{h}^{\alpha_{j}}\right) \subset \Omega_{\alpha_{j}}, x\left(\sup J_{h}^{\alpha_{j}}\right) \in \partial \Omega_{\alpha_{j}}$, which is not possible because of the transversality conditions (2.1). For simplicity of notations, by possibly renaming the indexes $\alpha_{i}$, we shall assume in the following that

$$
\alpha_{1}>\cdots>\alpha_{I} .
$$

Then, by induction on $m=0, \ldots, N_{I}$ we shall prove that there exist points $t_{0}=\tau+\delta>t_{1}>\cdots>t_{I}=\tau-\delta$ such that, if we set

$$
F_{\alpha_{i}} \doteq E_{\alpha_{i}} \backslash \bigcup_{i<j \leq I} E_{\alpha_{j}}
$$

one has

$$
F_{\alpha_{I-m}} \cap(\tau-\delta, \tau+\delta]=\left(t_{m+1}, t_{m}\right]
$$

which, recalling the Definition 2.2, yields the conclusion. Indeed, since

$$
(\tau-\delta, \tau+\delta] \subseteq \bigcup_{i=1}^{I} E_{\alpha_{i}}
$$

and because of

$$
\alpha^{*}(x(t)) \leq \alpha_{I} \quad \forall t \in(\tau-\delta, \tau+\delta]
$$


from (3.3) it clearly follows that $F_{\alpha_{I}} \cap(\tau-\delta, \tau+\delta]=E_{\alpha_{I}} \cap(\tau-\delta, \tau+\delta]$ must be equal to a single interval $\left(t_{1}, \tau+\delta\right]$, proving (3.4) in the case $m=0$. Next, assume to have established (3.4) for $m=0, \ldots, \bar{m}, \bar{m} \geq 0$. Then, one has

$$
\left(\tau, t_{\bar{m}+1}\right] \subset \bigcup_{i=1}^{I-\bar{m}-1} F_{\alpha_{i}} \subset \bigcup_{i=1}^{I-\bar{m}-1} E_{\alpha_{i}}
$$

and hence

$$
\alpha^{*}(x(t)) \leq \alpha_{I-\bar{m}-1} \quad \forall t \in\left(\tau, t_{\bar{m}+1}\right] .
$$

Thus, using again (3.3) we deduce that $F_{\alpha_{I-\bar{m}}} \cap(\tau, \tau+\delta]$ must be equal to a single interval $\left(t_{\bar{m}+2}, t_{\bar{m}+1}\right]$, proving the inductive step.

Concerning (ii), to prove the local existence of a forward Carathéodory solutions to (2.4), consider the index

$$
\bar{\alpha} \doteq \max \left\{\alpha: x_{0} \in \bar{\Omega}_{\alpha}\right\}
$$

Because of the transversality condition (2.1), the solution of the Cauchy problem

$$
\dot{x}=g_{\bar{\alpha}}(x), \quad x(0)=x_{0}
$$

remains inside $\Omega_{\bar{\alpha}}$ for all $t \geq 0$. Hence it provides also a solution of (2.4) on some positive interval $[0, \delta]$.

To show the backward uniqueness property, let $x_{1}(\cdot), x_{2}(\cdot)$ be any two Carathéodory solutions to (2.4) with $x_{1}(\tau)=x_{2}(\tau)=\bar{x}$. For $i=1,2$, call

$$
\alpha_{i}^{*}(t) \doteq \max \left\{\alpha: x_{i}(t) \in \Omega_{\alpha}\right\}
$$

By (i), the maps $t \mapsto \alpha_{i}^{*}(t)$ are piecewise constant, non-decreasing and left continuous. Hence there exists $\delta>0$ and indices $\alpha^{\prime}, a^{\prime \prime}$ such that

$$
\alpha_{1}^{*}(t)=\alpha^{\prime}, \quad \alpha_{2}^{*}(t)=\alpha^{\prime \prime} \quad \forall t \in[\tau-\delta, \tau] .
$$

But then one has

$$
\alpha^{\prime}=\alpha_{1}^{*}(\tau)=\alpha_{2}^{*}(\tau)=\alpha^{\prime \prime}
$$

The uniqueness of backward solutions is now clear, because on $[\tau-\delta, \tau]$ both $x_{1}$ and $x_{2}$ are solutions of the same Cauchy problem with smooth coefficients

$$
\dot{x}=g_{\alpha^{\prime}}(x), \quad x(\tau)=\bar{x} .
$$

To prove (iii), consider a sequence $\left(x_{n}(\cdot)\right)_{n}$ of Carathéodory solutions to (2.4) defined on some intervals $\left[a_{n}, b_{n}\right]$, so that (3.2) holds.

Since $\left\{\Omega_{\alpha}: \alpha \in \mathcal{A}\right\}$ is a locally finite covering and because of (3.5), we may assume that each $\left[a_{n}, b_{n}\right]$ intersects only a uniformly finite number of elements in $\left\{x_{n}^{-1}\left(\Omega_{\alpha}\right): \alpha \in \mathcal{A}\right\}$. Then, set

$$
\left\{\alpha_{1}, \ldots, \alpha_{I}\right\} \doteq\left\{\alpha \in \mathcal{A}:\left[a_{n}, b_{n}\right] \cap x_{n}^{-1}\left(\Omega_{\alpha}\right) \neq \emptyset \text { for some } n\right\}
$$

with

$$
\alpha_{1} \leq \cdots \leq \alpha_{I} .
$$

By (ii), for any $n \in \mathbb{N}$, let $t_{n, 0}=a \leq \cdots \leq t_{n, I}=b$ be an $I+1$-tuple of points in $\left[a_{n}, b_{n}\right]$ such that

$$
x_{n}(t) \in \Omega_{\alpha_{i}} \backslash \bigcup_{\beta>\alpha_{i}} \Omega_{\beta} \quad \forall t \in\left(t_{n, i-1}, t_{n, i}\right], \quad i=1, \ldots, I .
$$


The set $\left\{t \in\left[a_{n}, b_{n}\right]: x_{n}(t) \in \Omega_{\alpha_{i}} \backslash \bigcup_{\beta>\alpha_{i}} \Omega_{\beta}\right\}$ may well be empty for some $i=1, \ldots, I, n \in \mathbb{N}$, in which case one has $t_{n, i-1}=t_{n, i}$. Notice that, because of Definition 2.2, from (3.6) it follows

$$
x_{n}(t)=x_{n}(a)+\sum_{\ell=1}^{i-1} \int_{t_{n, \ell-1}}^{t_{n, \ell}} g_{\alpha_{\ell}}\left(x_{n}(s)\right) d s+\int_{t_{n, i-1}}^{t} g_{\alpha_{i}}\left(x_{n}(s)\right) d s \quad \forall t \in\left[t_{n, i-1}, t_{n, i}\right] .
$$

On the other hand, by possibly taking a subsequence, we may assume that any $\left(t_{n, i}\right)_{n}, i=0, \ldots, I$, converges in $[a, b]$ and set

$$
\hat{t}_{i} \doteq \lim _{n \rightarrow \infty} t_{n, i} \quad i=0, \ldots, I .
$$

Then, since

$$
\left(\hat{t}_{i-1}, \hat{t}_{i}\right)=\bigcup_{k=1}^{\infty} \bigcap_{n=k}^{\infty}\left(t_{n, i-1}, t_{n, i}\right]
$$

from (3.5-3.7) we deduce

$$
\begin{gathered}
\hat{x}(t) \in \bar{\Omega}_{\alpha_{i}} \backslash \bigcup_{\beta>\alpha_{i}} \Omega_{\beta}, \\
\hat{x}(t)=\hat{x}(a)+\sum_{\ell=1}^{i-1} \int_{\hat{t}_{\ell-1}}^{\hat{t}_{\ell}} g_{\alpha_{\ell}}(\hat{x}(s)) d s+\int_{\hat{t}_{i-1}}^{t} g_{\alpha_{i}}(\hat{x}(s)) d s
\end{gathered} \quad \forall t \in\left[\hat{t}_{i-1}, \hat{t}_{i}\right], \quad i=1, \ldots, I .
$$

This, in particular, means that $\hat{x}(\cdot)$ is the classical solution to

$$
\dot{x}=g_{\alpha_{i}}(x)
$$

on $\left[\hat{t}_{i-1}, \hat{t}_{i}\right]$, and that

$$
\dot{\hat{x}}(s-)=g_{\alpha_{i}}(x(s)) \quad \forall s \in\left(\hat{t}_{i-1}, \hat{t}_{i}\right] .
$$

Moreover observe that, because of the transversality condition (2.1), the set $\left\{t \in\left[\hat{t}_{i-1}, \hat{t}_{i}\right]: \hat{x}(t) \in \partial \Omega_{\alpha_{i}}\right\}$ is nowhere dense in $\left[\hat{t}_{i-1}, \hat{t}_{i}\right]$. Thus, if $s$ is any point in $\left(\hat{t}_{i-1}, \hat{t}_{i}\right]$ such that $\hat{x}(s) \in \partial \Omega_{\alpha_{i}}$, there will be some increasing sequence $\left(s_{n}\right)_{n} \subset\left(\hat{t}_{i-1}, \hat{t}_{i}\right)$ converging to $s$ and such that $\hat{x}\left(s_{n}\right) \in \Omega_{\alpha_{i}}$. But this yields a contradiction to $(2.1)$ since then

$$
0 \leq \lim _{n \rightarrow \infty}\left\langle\frac{\hat{x}(s)-\hat{x}\left(s_{n}\right)}{s-s_{n}}, \mathbf{n}(\hat{x}(s))\right\rangle=\langle\dot{\hat{x}}(s-), \mathbf{n}(\hat{x}(s))\rangle=\left\langle g_{\alpha_{i}}(\hat{x}(s)), \mathbf{n}(\hat{x}(s))\right\rangle .
$$

Hence, recalling the definition (2.2), from (3.8) we derive

$$
\begin{gathered}
\hat{x}(t) \in \Omega_{\alpha_{i}} \backslash \bigcup_{\beta>\alpha_{i}} \Omega_{\beta} \quad \forall t \in\left(\hat{t}_{i-1}, \hat{t}_{i}\right], \quad i=1, \ldots, I, \\
\hat{x}(t)=\hat{x}(a)+\int_{a}^{t} g(\hat{x}(s)) d s \quad \forall t \in[a, b],
\end{gathered}
$$

proving that $\hat{x}:[a, b] \mapsto \Omega$ is the Carathéodory solution to $(2.4)$ on $[a, b]$.

Concerning (iv), consider now a sequence $x_{\pi_{n}}:[a, b] \mapsto \Omega$ of forward Euler $\pi$-solutions to (2.4) associated with partitions

$$
\pi_{n}=\left\{\tilde{t}_{n, 0}, \ldots, \tilde{t}_{n, N_{\pi_{n}}}\right\}
$$


having initial error $\eta_{\pi_{n}}$ and discrete external disturbance

$$
\left\{c_{n, 0}, \ldots, c_{n, N_{n}}\right\}
$$

Assume that maximal mesh size $\delta_{\pi_{n}}=\sup _{i}\left(\tilde{t}_{n, i+1}-\tilde{t}_{n, i}\right)$, initial measurement error $\eta_{\pi_{n}}$ and external disturbance $\zeta_{\pi_{n}}=\sup _{i}\left|c_{n, i}\right|$ are such that

$$
\lim _{n \rightarrow \infty} \delta_{\pi_{n}}=0, \quad \lim _{n \rightarrow \infty} \eta_{\pi_{n}}=0, \quad \lim _{n \rightarrow \infty} \zeta_{\pi_{n}}=0,
$$

and that $\left(x_{\pi_{n}}\right)_{n}$ converges uniformly on $[a, b]$, as $n \rightarrow \infty$, to some function $\hat{x}:[a, b] \mapsto \Omega$. Here, it is not restrictive to suppose $\Omega$ to be bounded since, otherwise, one can clearly replace it with a neighborhood of $\hat{x}([a, b])$ that contains all the sets $x_{\pi_{n}}([a, b])$, for $n$ sufficiently large. Observe that, since $\left\{\Omega_{\alpha}: \alpha \in \mathcal{A}\right\}$ is a locally finite covering of $\Omega$, the interval $[a, b]$ intersects only a uniformly finite number of elements in $\left\{x_{\pi_{n}}^{-1}\left(\Omega_{\alpha}\right): \alpha \in \mathcal{A}\right\}$. Then, set

$$
\left\{\alpha_{1}, \ldots, \alpha_{I}\right\} \doteq\left\{\alpha \in \mathcal{A}: \quad \exists n \text { s.t. } \quad[a, b] \cap x_{\pi_{n}}^{-1}\left(\Omega_{\alpha}\right) \neq \emptyset\right\}
$$

with

$$
\alpha_{1} \leq \cdots \leq \alpha_{I}
$$

Moreover, because each field $g_{\alpha_{i}}$ is smooth on $\bar{\Omega}_{\alpha_{i}}$ and satisfies condition (2.1) at the boundary $\partial \Omega_{\alpha_{i}}$, one can choose $0<\rho$ and find some constants $L, C>0$, so that

$$
\begin{gathered}
\sup _{\substack{x \in B\left(\bar{\Omega}_{\alpha_{i}}, \rho\right) \\
i \in\{1, \ldots, I\}}}\left|g_{\alpha_{i}}(x)\right| \leq L, \quad \sup _{\substack{x \in B\left(\bar{\Omega}_{\alpha_{i}}, \rho\right) \\
i \in\{1, \ldots, I\}}}\left|D g_{\alpha_{i}}(x)\right| \leq C, \\
c_{\rho} \doteq \sup \left\{\left\langle g_{\alpha_{i}}(y), \mathbf{n}(x)\right\rangle: x \in \partial \Omega_{\alpha_{i}}, \quad y \in B(x, \rho) \cap \Omega_{\alpha_{i}}, \quad i=1, \ldots, I\right\}<0 .
\end{gathered}
$$

The bound (3.10), in particular, implies that if $\eta_{\pi_{n}}<\rho$ one has

$$
\left|x_{\pi_{n}}(t)-x_{\pi_{n}}(s)\right| \leq\left(L+\zeta_{\pi_{n}}\right) \delta_{\pi_{n}} \quad \forall t, s \in\left[\tilde{t}_{n, m-1}, \tilde{t}_{n, m}\right], \quad 1 \leq m \leq N_{\pi_{n}} .
$$

Hence, from (3.11-3.12) we deduce that, if $n$ is large enough so that $\delta_{\pi_{n}}, \alpha_{\pi_{n}}, \eta_{\pi_{n}}$ are sufficiently small, for all $m=1, \ldots, N_{\pi_{n}}$ there holds

$$
\begin{gathered}
\left|x_{\pi_{n}}\left(\tilde{t}_{n, m}\right)-x_{\pi_{n}}\left(\tilde{t}_{n, m-1}\right)\right|<\rho / 2 \\
x_{\pi_{n}}\left(\tilde{t}_{n, m-1}\right) \in \Omega_{\alpha_{i}} \backslash \bigcup_{\beta>\alpha_{i}} \Omega_{\beta} \Longrightarrow \quad x_{\pi_{n}}\left(\tilde{t}_{n, m}\right) \in \bigcup_{\beta \geq \alpha_{i}} \Omega_{\beta} .
\end{gathered}
$$

Therefore, as in the proof of (iii), using (3.13) we find, for any $n$, an $I+1$-tuple of points $t_{n, 0}=a \leq \cdots \leq t_{n, I}=b$, such that

$$
x_{\pi_{n}}\left(\tilde{t}_{n, m}\right) \in \Omega_{\alpha_{i}} \backslash \bigcup_{\beta>\alpha_{i}} \Omega_{\beta} \quad \forall \tilde{t}_{n, m} \in\left(t_{n, i-1}, t_{n, i}\right], \quad i=1, \ldots, I .
$$

By possibly taking a subsequence, each $\left(t_{n, i}\right)_{n}$ will converge in $[a, b]$. We can thus set

$$
\hat{t}_{i} \doteq \lim _{n \rightarrow \infty} t_{n, i} \quad i=0, \ldots, I .
$$


Hence, from (3.14) we deduce

$$
\hat{x}(t) \in \bar{\Omega}_{\alpha_{i}} \backslash \bigcup_{\beta>\alpha_{i}} \Omega_{\beta}, \quad \forall t \in\left[\hat{t}_{i-1}, \hat{t}_{i}\right], \quad i=1, \ldots, I
$$

On the other hand observe that, if $\bar{s}$ is any point in $\left(\hat{t}_{i-1}, \hat{t}_{i}\right)$ such that $\hat{x}(\bar{s}) \in \partial \Omega_{\alpha_{i}}$, one can find an increasing sequence of partition points $\tilde{t}_{n, m_{n}} \in\left(t_{n, i-1}, t_{n, i}\right)$ of $\pi_{n}$ converging to $\bar{s}$ slowly enough so that

$$
\limsup _{n \rightarrow \infty} \frac{\hat{x}(\bar{s})-x_{\pi_{n}}\left(\tilde{t}_{n, m_{n}}\right)}{\bar{s}-\tilde{t}_{n, m_{n}}}=\limsup _{n \rightarrow \infty} \frac{x_{\pi_{n}}(\bar{s})-x_{\pi_{n}}\left(\tilde{t}_{n, m_{n}}\right)}{\bar{s}-\tilde{t}_{n, m_{n}}}
$$

Moreover, for $n$ large enough, one can assume that

$$
\begin{aligned}
\left(\tilde{t}_{n, m_{n}}, \bar{s}\right) & \subset\left(t_{n, i-1}, t_{n, i}\right), & & \\
\mid\left\langle c_{n, \ell}, \mathbf{n}(\hat{x}(\bar{s})\rangle\right| & <\left|c_{\rho}\right| / 2 & & \forall \ell, \\
\left|x_{\pi_{n}}(t)-\hat{x}(\bar{s})\right| & <\rho & & \forall t \in\left[\tilde{t}_{n, m_{n}}, \bar{s}\right],
\end{aligned}
$$

where $\rho, c_{\rho}$, are as in (3.10-3.11). But then, letting

$$
\tilde{t}_{n, m_{n}}<\cdots<\tilde{t}_{n, m_{p}} \leq \bar{s}
$$

denote the partition points of $\pi_{n}$ lying between $\tilde{t}_{n, m_{n}}$ and $\bar{s}$, setting $\tilde{t}_{n, m_{p}+1} \doteq \bar{s}$ and using (3.12), one would derive a contradiction since

$$
\begin{aligned}
0 & \leq \limsup _{n \rightarrow \infty}\left\langle\frac{\hat{x}(\bar{s})-x_{\pi_{n}}\left(\tilde{t}_{n, m_{n}}\right)}{\bar{s}-\tilde{t}_{n, m_{n}}}, \mathbf{n}(\hat{x}(\bar{s}))\right\rangle=\limsup _{n \rightarrow \infty}\left\langle\frac{x_{\pi_{n}}(\bar{s})-x_{\pi_{n}}\left(\tilde{t}_{n, m_{n}}\right)}{\bar{s}-\tilde{t}_{n, m_{n}}}, \mathbf{n}(\hat{x}(\bar{s}))\right\rangle \\
& =\limsup _{n \rightarrow \infty}\left(\tilde{t}_{n, m_{p}+1}-\tilde{t}_{n, m_{n}}\right)^{-1} \sum_{\ell=m_{n}}^{m_{p}}\left(\tilde{t}_{n, \ell+1}-\tilde{t}_{n, \ell}\right)\left\langle g_{\alpha_{i}}\left(x_{\pi_{n}}\left(\tilde{t}_{n, \ell}\right)\right)+c_{n, \ell}, \mathbf{n}(\hat{x}(\bar{s}))\right\rangle \leq-\left|c_{\rho}\right| / 2 .
\end{aligned}
$$

Whence, by (3.15), it must be

$$
\hat{x}(t) \in \Omega_{\alpha_{i}} \backslash \bigcup_{\beta>\alpha_{i}} \Omega_{\beta}, \quad \forall t \in\left(\hat{t}_{i-1}, \hat{t}_{i}\right), \quad i=1, \ldots, I .
$$

Next, set

$$
J_{n} \doteq \bigcup_{\left\{1 \leq i \leq I: \hat{t}_{i-1} \neq \hat{t}_{i}\right\}}\left(t_{n, i-1}, t_{n, i}\right]
$$

and, since $x_{\pi_{n}}\left(t_{n, i}\right) \rightarrow \hat{x}\left(\hat{t}_{i}\right)$, let $n$ be large enough so that

$$
\delta_{\pi_{n}} \ll \inf \left\{\left(t_{n, i}-t_{n, i-1}\right): \hat{t}_{i-1} \neq \hat{t}_{i}, \quad 1 \leq i \leq I\right\}
$$

Then, if $t_{n, i}$ is a point satisfying (3.14) such that $\hat{t}_{i-1} \neq \hat{t}_{i}$, and $\tilde{t}_{n, \ell}$ denotes any partition point of $\pi_{n}$ in $\left(t_{n, i-1}, t_{n, i}\right)$, one has

$$
\left|x_{\pi_{n}}\left(\tilde{t}_{n, \ell}\right)-x_{\pi_{n}}(t)\right| \leq\left(L+\zeta_{\pi_{n}}\right) \delta_{\pi_{n}} \quad \forall t \in\left(t_{n, i-1}+\delta_{\pi_{n}}, t_{n, i}\right) \cap\left(\tilde{t}_{n, \ell}, \tilde{t}_{n, \ell+1}\right) .
$$


Thus, there follows

$$
\begin{aligned}
\left|\dot{x}_{\pi_{n}}(t)-g_{\alpha_{i}}\left(x_{\pi_{n}}(t)\right)\right|= & \left|g\left(x_{\pi_{n}}(t)\right)+c_{n, \ell}-g_{\alpha_{i}}\left(x_{\pi_{n}}(t)\right)\right|=\left|g_{\alpha_{i}}\left(x_{\pi_{n}}\left(\tilde{t}_{n, \ell}\right)\right)+c_{n, \ell}-g_{\alpha_{i}}\left(x_{\pi_{n}}(t)\right)\right| \\
& \leq\left|g_{\alpha_{i}}\left(x_{\pi_{n}}\left(\tilde{t}_{n, \ell}\right)\right)-g_{\alpha_{i}}\left(x_{\pi_{n}}(t)\right)\right|+\zeta_{\pi_{n}} \\
& <C\left(L+\zeta_{\pi_{n}}\right) \delta_{\pi_{n}}+\zeta_{\pi_{n}}, \quad \forall t \in\left(t_{n, i-1}+\delta_{\pi_{n}}, t_{n, i}\right) .
\end{aligned}
$$

Hence, for any $i=1, \ldots I$, and any $t \in[a, b] \cap\left[t_{n, i-1}, t_{n, i}\right]$, if $n$ is large enough so that $\eta_{\pi_{n}}<\rho$ with $\rho$ as in (3.10), we have

$$
\begin{aligned}
\left|x_{\pi_{n}}(t)-x_{\pi_{n}}(a)-\int_{a}^{t} g\left(x_{\pi_{n}}(s)\right) d s\right| \leq & \sum_{j=1}^{i-1} \int_{t_{n, j-1}+\delta_{\pi_{n}}}^{t_{n, j}}\left|\dot{x}_{\pi_{n}}(s)-g_{\alpha_{j}}\left(x_{\pi_{n}}(s)\right)\right| d s \\
& +\int_{t_{n, i-1}+\delta_{\pi_{n}}}^{t}\left|\dot{x}_{\pi_{n}}(s)-g_{\alpha_{i}}\left(x_{\pi_{n}}(s)\right)\right| d s+\left(I \delta_{\pi_{n}}+\left|J_{n}\right|\right) 2 L \\
\leq & (t-a)\left[C\left(L+\zeta_{\pi_{n}}\right) \delta_{\pi_{n}}+\zeta_{\pi_{n}}\right]+\left(I \delta_{\pi_{n}}+\left|J_{n}\right|\right) 2 L .
\end{aligned}
$$

Letting $n \rightarrow \infty$ in (3.18), since $\zeta_{\pi_{n}}, \delta_{\pi_{n}},\left|J_{n}\right| \rightarrow 0$, we obtain

$$
\left|\hat{x}(t)-\hat{x}(a)-\int_{a}^{t} g(\hat{x}(s)) d s\right|=0 \quad \forall t \in[a, b]
$$

proving that $\hat{x}(\cdot)$ is the Carathéodory solution to $(2.4)$ on $[a, b]$.

Assume now that a given function $\hat{x}:[a, b] \mapsto \Omega$ is a Carathéodory solution of $(2.4)$ on $[a, b]$. We shall construct a sequence $\left(x_{\pi_{n}}\right)_{n}$ of forward Euler $\pi$-solutions to (2.4) perturbed by some external disturbance which converge, uniformly on $[a, b]$, to $\hat{x}$ as mesh size and external disturbance both tend to zero. Let

$$
\left\{\alpha_{1}, \ldots, \alpha_{I}\right\} \doteq\left\{\alpha \in \mathcal{A}:[a, b] \cap \hat{x}^{-1}\left(\Omega_{\alpha}\right) \neq \emptyset\right\}
$$

with

$$
\alpha_{1}<\cdots<\alpha_{I}
$$

By (ii), denote $\hat{t}_{0}=a<\cdots<\hat{t}_{I}=b$ an $I+1$-tuple of points in $[a, b]$ such that

$$
\hat{x}(t) \in \Omega_{\alpha_{i}} \backslash \bigcup_{\beta>\alpha_{i}} \Omega_{\beta} \quad \forall t \in\left(\hat{t}_{i-1}, \hat{t}_{i}\right], \quad i=1, \ldots, I .
$$

Next, for any $n \in \mathbb{N}$, define the partition $\pi_{n}=\left\{\tilde{t}_{n, \ell} ; 0 \leq \ell \leq n I\right\}$ by setting

$$
\tilde{t}_{n, 0} \doteq a, \quad \tilde{t}_{n, n i+m} \doteq \tilde{t}_{n, n i}+\frac{m}{n}\left(\hat{t}_{i+1}-\hat{t}_{i}\right) \quad i=0, \ldots, I-1, \quad m=1, \ldots, n
$$

Then, since $\hat{x}(\cdot)$ is the classical solution to

$$
\dot{x}=g_{\alpha_{i}}(x)
$$

on $\left[\hat{t}_{i-1}, \hat{t}_{i}\right]$, for every $m=1, \ldots, n$, choose $s_{n, n i+m} \in\left(\tilde{t}_{n, n i+m}, \tilde{t}_{n, n i+m+1}\right)$ such that

$$
g_{\alpha_{i+1}}\left(\hat{x}\left(s_{n, n i+m}\right)\right)=\dot{\hat{x}}\left(s_{n, n i+m}\right)=\frac{\hat{x}\left(\tilde{t}_{n, n i+m+1}\right)-\hat{x}\left(\tilde{t}_{n, n i+m}\right)}{\tilde{t}_{n, n i+m+1}-\tilde{t}_{n, n i+m}} .
$$


Moreover, by (3.19), $\hat{x}(a) \in \overline{\Omega_{\alpha_{1}} \backslash \bigcup_{\beta>\alpha_{1}} \Omega_{\beta}}$ and hence there will be a sequence $\left(e_{n}\right)_{n}$ in $\mathbb{R}^{n}$, with $\eta_{n} \doteq\left|e_{n}\right| \rightarrow 0$ as $n \rightarrow \infty$, such that

$$
\hat{x}(a)+e_{n} \in \Omega_{\alpha_{1}} \backslash \bigcup_{\beta>\alpha_{1}} \Omega_{\beta} \quad \forall n .
$$

In connection with the partition $\pi_{n}$, let $x_{\pi_{n}}$ be the forward Euler $\pi$-solution to (2.4) perturbed by the discrete external disturbance

$$
c_{n, n i+m} \doteq g_{\alpha_{i+1}}\left(\hat{x}\left(s_{n, n i+m}\right)\right)-g_{\alpha_{i+1}}\left(\hat{x}\left(\tilde{t}_{n, n i+m}\right)\right) \quad i=0, \ldots, I-1, \quad m=1, \ldots, n,
$$

with initial measurement error $e_{n}$ as above, and with initial condition $x_{\pi_{n}}(a)=\hat{x}(a)$. Notice that $\zeta_{\pi_{n}} \doteq$ $\sup _{i, m}\left|c_{n, n i+m}\right| \rightarrow 0$ as $n \rightarrow \infty$, and $x_{\pi_{n}}$ is recursively defined by

$$
x_{\pi_{n}}(t)=\left\{\begin{array}{lr}
\hat{x}(a)+g\left(\hat{x}(a)+e_{n}\right)(t-a) & \text { if } \quad t \in\left[a, \tilde{t}_{n, 1}\right], \\
x_{\pi_{n}}\left(\tilde{t}_{n, n i+m}\right)+\frac{\hat{x}\left(\tilde{t}_{n, n i+m+1}\right)-\hat{x}\left(\tilde{t}_{n, n i+m}\right)}{\tilde{t}_{n, n i+m+1}-\tilde{t}_{n, n i+m}}\left(t-\tilde{t}_{n, n i+m}\right) & \text { if } \quad t \in\left[\tilde{t}_{n, n i+m}, \tilde{t}_{n, n i+m+1}\right] \\
n i+m>0,
\end{array}\right.
$$

i.e., $x_{\pi_{n}}$ is precisely the polygonal function with vertices at the points $\hat{x}\left(\tilde{t}_{n, \ell}\right), 0 \leq \ell \leq n I$, of $\hat{x}$. Such polygonals converge uniformly to $\hat{x}$ as mesh size

$$
\delta_{\pi_{n}} \doteq \frac{1}{n}\left[\sup _{0 \leq i \leq I-1}\left|\hat{t}_{i+1}-\hat{t}_{i}\right|\right]
$$

tends to zero. Indeed, for $n$ sufficiently large, we can assume that

$$
x_{\pi_{n}}(t) \in \bar{\Omega}_{\alpha_{i}} \quad \forall t \in\left[\hat{t}_{i-1}, \hat{t}_{i}\right], \quad i=1, \ldots, I,
$$

and hence, letting $C, L>0$ be some constant such that

$$
\sup _{\substack{x \in \bar{\Omega}_{\alpha_{i}} \\ i \in\{1, \ldots, I\}}}\left|g_{\alpha_{i}}(x)\right| \leq L, \quad \sup _{\substack{x \in \bar{\Omega}_{\alpha_{i}} \\ i \in\{1, \ldots, I\}}}\left|D g_{\alpha_{i}}(x)\right| \leq C,
$$

from

we deduce

$$
\begin{array}{lll}
\dot{x}_{\pi_{n}}(t)=g_{\alpha_{1}}\left(\hat{x}(a)+e_{n}\right) & t \in\left[a, \tilde{t}_{n, 1}\right], & \\
\dot{x}_{\pi_{n}}(t)=g_{\alpha_{i+1}}\left(\hat{x}\left(s_{n, n i+m}\right)\right) & t \in\left(\tilde{t}_{n, n i+m}, \tilde{t}_{n, n i+m+1}\right) & n i+m>0,
\end{array}
$$

thus concluding the proof.

$$
\left|x_{\pi_{n}}(t)-\hat{x}(t)\right| \leq \int_{a}^{t}\left|\dot{x}_{\pi_{n}}(s)-\dot{\hat{x}}(s)\right| d s \leq\left[(b-a) L+\eta_{n}\right] C \delta_{\pi_{n}}
$$

An Example. Consider the covering of $\Omega \doteq \mathbb{R}^{2}$ consisting of

$$
\begin{aligned}
& \Omega_{1} \doteq \Omega, \\
& \Omega_{2} \doteq\left\{\left(x_{1}, x_{2}\right) \in \Omega: x_{2}<-x_{1}^{2}\right\}, \\
& \Omega_{3} \doteq\left\{\left(x_{1}, x_{2}\right) \in \Omega: x_{2}>x_{1}^{2}\right\},
\end{aligned}
$$




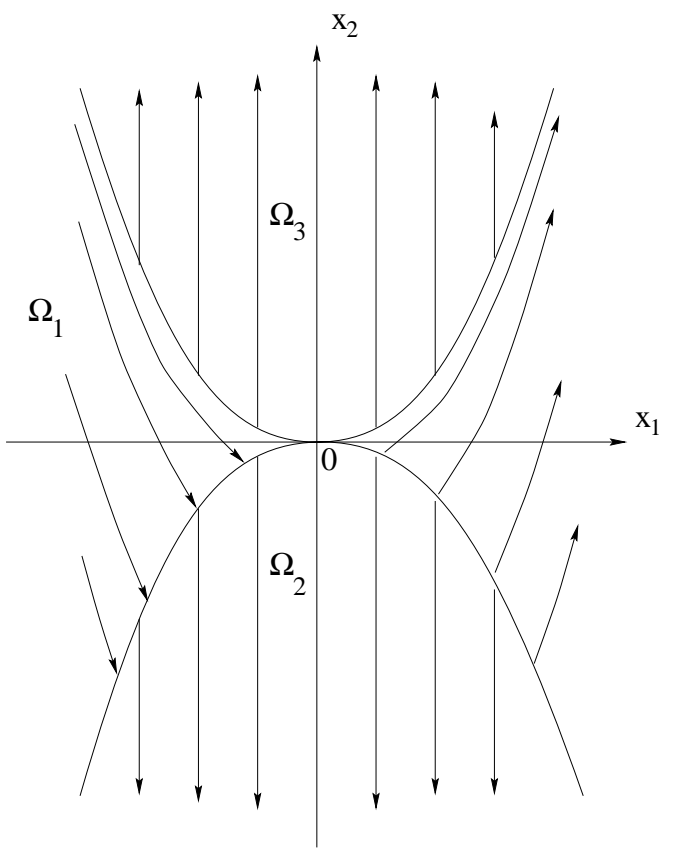

FiguRE 1

and the family of inward-pointing vector fields

$$
\begin{array}{ll}
g_{1}: \Omega_{1} \rightarrow \mathbb{R}^{2}, & f_{1}\left(x_{1}, x_{2}\right) \doteq\left(1,2 x_{1}\right), \\
g_{2}: \Omega_{2} \rightarrow \mathbb{R}^{2}, & f_{2}\left(x_{1}, x_{2}\right) \doteq(0,-1), \\
g_{3}: \Omega_{3} \rightarrow \mathbb{R}^{2}, & f_{3}\left(x_{1}, x_{2}\right) \doteq(0,1) .
\end{array}
$$

Then, the vector field $g$ on $\Omega$ defined by

$$
g\left(x_{1}, x_{2}\right)=\left\{\begin{array}{lll}
\left(1,2 x_{1}\right) & \text { if } & \left|x_{2}\right| \leq x_{1}^{2} \\
(0,-1) & \text { if } & x_{2}<-x_{1}^{2} \\
(0,1) & \text { if } & x_{2}>x_{1}^{2}
\end{array}\right.
$$

is the patchy vector field associated with $\left\{\Omega_{\alpha}: \alpha=1,2,3\right\}$ and $\left\{g_{\alpha}: \alpha=1,2,3\right\}$. We shall compare now the set of Carathéodory solutions to the Cauchy problem

$$
\dot{x}=g(x), \quad x(0)=(-1,1),
$$

with the sets of various other types of generalized solutions considered in the literature.

1. Consider the set $S_{C}^{\max }$ of maximal forward Carathéodory solutions to $(3.23)$. Let $\tilde{x}:[0, \infty) \mapsto \Omega$ be the map defined by

$$
\tilde{x}(t) \doteq\left\{\begin{array}{lll}
\left(-1+t, 1-2 t+t^{2}\right) & \text { if } & 0 \leq t \leq 1 \\
(0,1-t) & \text { if } & t \geq 1,
\end{array}\right.
$$


and, for any fixed $s \in \mathbb{R}^{+}$, define the maps

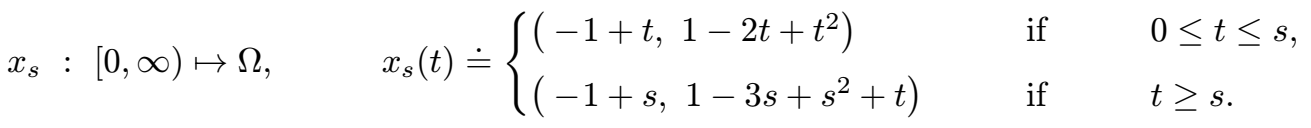

Then one has

$$
S_{C}^{\max }=\{\tilde{x}\} \cup\left\{x_{s}: 0 \leq s \leq \infty\right\}
$$

2. Consider the set $S_{E}^{\max }$ of uniform limits of forward Euler-solutions to (3.23) without any (initial statemeasurement or external) perturbation, defined on $[0, \infty)$, i.e. the set of functions that are uniform limits of some sequence of polygonal maps recursively defined by

$$
x_{\pi}(0)=(-1,1), \quad x_{\pi}(t)=x_{\pi}\left(\tilde{t}_{i}\right)+g\left(x_{\pi}\left(\tilde{t}_{i}\right)\right)\left(t-\tilde{t}_{i}\right) \quad t \in\left[\tilde{t}_{i}, \tilde{t}_{i+1}\right],
$$

in connection with partitions $\pi_{n}=\left\{\tilde{t}_{n, 0}, \tilde{t}_{n, 1}, \ldots, \tilde{t}_{n, N_{\pi_{n}}}\right\}$ of $[0, \infty)$ having mesh size $\delta_{\pi_{n}} \rightarrow 0$. Then one has

with $\tilde{x}$ defined as in (3.24).

$$
S_{E}^{\max }=\{\tilde{x}\} \cup\left\{x_{\infty}\right\}
$$

3. Consider the set $S_{S}^{\max }$ of uniform limits of sampling-solutions to $(3.23)$ on $[0, \infty)$, i.e. the set of functions that are uniform limits of some sequence of piecewise smooth maps recursively obtained by solving

$$
\dot{x}_{\pi}(t)=g_{\alpha^{*}\left(x_{\pi}\left(\tilde{t}_{i}\right)\right)}\left(x_{\pi}(t)\right) \quad t \in\left[\tilde{t}_{i}, \tilde{t}_{i+1}\right]
$$

using as initial condition $x_{\pi}\left(\tilde{t}_{i}\right)$ the endpoint of the solution on the preceding interval (and starting with $\left.x_{\pi}(0)=(-1,1)\right)$, in connection with partitions $\pi_{n}=\left\{\tilde{t}_{n, 0}, \tilde{t}_{n, 1}, \ldots, \tilde{t}_{n, N_{\pi_{n}}}\right\}$ of $[0$, $\infty)$ having mesh size $\delta_{\pi_{n}} \rightarrow 0$. Then one has

with $x_{\infty}$ defined as in $(3.25)$

$$
S_{S}^{\max }=\left\{x_{\infty}\right\}
$$

4. Consider the set $S_{F}^{\max }$ of Filippov solutions to $(3.23)$ on $[0, \infty)$, i.e. the set of absolutely continuous function $x:[0, \infty) \mapsto \Omega$ that satisfy

with

$$
\dot{x}(t) \in F(x(t)) \quad \text { a.e. } t>0
$$

$$
F(x)=\bigcap_{\delta>0} \bigcap_{|\mathcal{N}|=0} \overline{\operatorname{co}} g(B(x, \delta) \backslash \mathcal{N}),
$$

where $\overline{\mathrm{co}}$ denotes the closed convex hull. For any fixed $1 \leq r \leq \infty$, define the maps

$$
\begin{aligned}
y_{r}:[0, \infty) \mapsto \Omega, & y_{r}(t) \doteq\left\{\begin{array}{lll}
\left(-1+t, 1-2 t+t^{2}\right) & \text { if } & 0 \leq t \leq 1, \\
(0,0) & \text { if } & 1 \leq t \leq r, \\
(0, r-t) & \text { if } & t \geq r,
\end{array}\right. \\
z_{r}:[0, \infty) \mapsto \Omega, & z_{r}(t) \doteq\left\{\begin{array}{lll}
\left(-1+t, 1-2 t+t^{2}\right) & \text { if } & 0 \leq t \leq 1, \\
(0,0) & \text { if } & 1 \leq t \leq r, \\
(0,-r+t) & \text { if } & t \geq r .
\end{array}\right.
\end{aligned}
$$

Then one has

$$
S_{F}^{\max }=\{\tilde{x}\} \cup\left\{x_{s}: 0 \leq s \leq \infty\right\} \cup\left\{y_{r}: 1 \leq r \leq \infty\right\} \cup\left\{z_{r}: 1 \leq r \leq \infty\right\}
$$


with $\tilde{x}, x_{s}, x_{\infty}, y_{r}, z_{r}$, defined as in (3.24-3.26).

We remark that the set $S_{C}^{\max }$ of Carthéodory solutions is disconnected, being the union of the two disjoint sets $\{\tilde{x}\}$ and $\left\{x_{s}: 0 \leq s \leq \infty\right\}$.

The next Proposition provides analogous properties of those given by Proposition 3.1, for the trajectories of patchy vector fields subject to small external perturbations.

Proposition 3.2. Let $\chi: \Omega \mapsto R^{+}$be a continuous map, and $G: \Omega \mapsto 2^{\mathbb{R}^{n}}$ an admissible multivalued perturbation of a smooth patchy vector field $\left(\Omega, g,\left(\Omega_{\alpha}, g_{\alpha}\right)_{\alpha \in \mathcal{A}}\right)$ defined by

$$
G(x) \doteq g(x)+B(0, \chi(x)) \quad x \in \Omega \text {. }
$$

Then the following holds.

(i) If $t \mapsto x(t)$ is a Carathéodory solution to

$$
\dot{x} \in G(x),
$$

then the map $t \mapsto \alpha^{*}(t) \doteq \max \left\{\alpha: x(t) \in \Omega_{\alpha}\right\}$ is non-decreasing and left continuous.

(ii) For each $x_{0} \in \Omega, t_{0} \in \mathbb{R}$, the Cauchy problem (3.28) with initial condition $x\left(t_{0}\right)=x_{0}$ has at least one local forward Carathéodory solution.

(iii) The set of Carathéodory solutions of (3.28) is closed in the topology of uniform convergence.

The proof is entirely similar to the one of Proposition 3.1.

\section{Stabilization By PATCHY FEEDBACKS}

Toward the construction of a piecewise constant feedback which asymptotically stabilize a given asymptotically controllable system, we first establish two intermediate results.

Proposition 4.1. Let system (1.1) be globally asymptotically controllable to the origin. Then, for every $0<r<s$ there exist $T=T(r, s)>0, R=R(r, s)>0, \chi=\chi(r, s)>0$, and a patchy feedback control $U=U^{r, s}: D^{r, s} \mapsto K$ defined on some domain $D^{r, s}$ satisfying

$$
B(0, s) \backslash \circ \rightarrow B(0, r) \subset D^{r, s} \subset B(0, R),
$$

such that, for any measurable map $\zeta:[0, \infty) \mapsto \mathbb{R}^{n}$ with

$$
|\zeta(t)| \leq \chi \quad \text { for a.e. } t>0,
$$

and for any initial state $x_{0} \in D^{r, s} \backslash \stackrel{\circ}{B}(0, r)$, the perturbed system (2.17) admits a Carathéodory trajectory starting from $x_{0}$. Moreover, for any such trajectory $\gamma(\cdot)$, there exists $t_{\gamma} \leq T, t_{\gamma}<\tau^{\max }(\gamma)$, such that

$$
\left|\gamma\left(t_{\gamma}\right)\right|<r
$$


Proof. The proof is given in three steps.

Step 1. Fix $0<r<s$. Since (1.1) is globally asymptotically controllable and because the set of piecewise constant admissible controls is dense in the set of all admissible controls, for each $x_{0} \in B(0, s)$ we can find a piecewise constant admissible control $u_{0}=u_{x_{0}}$ and some constant $T_{0}=T_{x_{0}}>0$ such that

$$
\left|x\left(T_{0} ; x_{0}, u_{0}\right)\right|<r / 2 \text {. }
$$

Moreover, by possibly redefining $u_{0}$, we may assume that $\gamma_{0}(\cdot) \doteq x\left(\cdot ; x_{0}, u_{0}\right)$ takes different values at any two different poins $t, t^{\prime} \in\left[0, T_{0}\right]$. Let $t_{0,0}=0<t_{0,1}<\cdots<t_{0, N_{0}}=T_{0}$ be the points of discontinuity for $u_{0}$ on $\left[0, T_{0}\right]$ and $k_{0, j} \in K$ the corresponding values of $u_{0}$, i.e.

$$
u_{0}(t)=k_{0, j} \quad \text { if } \quad t \in\left(t_{0, j-1}, t_{0, j}\right), \quad j=1, \ldots, N_{0} .
$$

Set

$$
M_{0}=M_{x_{0}} \doteq \sup _{t \in\left[0, T_{0}\right]}\left|\gamma_{0}(t)\right|
$$

By the regularity of $f$ and the compactness of the set $K$ of admissible control values, there exists some constant $c_{0}=c_{x_{0}}>0$ such that, for any fixed $\tau \in\left[0, T_{0}\right]$, any initial point $\bar{x} \in B\left(\gamma_{0}(\tau), \rho\right), \rho>0$, and any Carathéodory trajectory $\gamma_{\rho, \chi}(\cdot), \chi>0$, of

$$
\dot{x}=f\left(x, u_{0}(t)\right)+B(0, \chi)
$$

starting from $\bar{x}$ at time $t=\tau$, there holds

$$
\sup _{t \in\left[\tau, T_{0}+\rho\right]}\left|\gamma_{\rho, \chi}(t)-\gamma_{0}(t)\right|<c_{0}(\rho+\chi) \quad \rho, \chi>0
$$

Thus, one can inductively deduce that for any fixed $j=1, \ldots N_{0}-1$, if

$$
\bar{x} \in B\left(\gamma_{0}\left(t_{0, j}\right), \sum_{k=2}^{j} c_{0}^{k-1} \chi+c_{0}^{j}(\rho+\chi)\right) \quad \rho>0
$$

and let $\gamma_{\rho, \chi}(\cdot), \chi>0$, be any Carathéodory trajectory of $(4.6)_{\chi}$ starting from $\bar{x}$ at time $t=t_{0, j}$, then one has

$$
\sup _{t \in\left[t_{0, j}, T_{0}+\rho\right]}\left|\gamma_{\rho, \chi}(t)-\gamma_{0}(t)\right|<\sum_{k=2}^{j+1} c_{0}^{k-1} \chi+c_{0}^{j+1}(\rho+\chi) \quad \rho, \chi>0 .
$$

Choose $\rho_{0}=\rho_{x_{0}}>0, \chi_{0}=\chi_{x_{0}}>0$ such that

$$
\sum_{k=2}^{N_{0}} c_{0}^{k-1} 2 \chi_{0}+c_{0}^{N_{0}}\left(\rho_{0}+2 \chi_{0}\right)<r / 2
$$

and set

$$
\rho_{x_{0}, 1}=\rho_{0,1} \doteq \rho_{0}, \quad \rho_{x_{0}, j}=\rho_{0, j} \doteq \sum_{k=3}^{j} c_{0}^{k-2} 2 \chi_{0}+c_{0}^{j-1}\left(\rho_{0}+2 \chi_{0}\right) \quad j=2, \ldots N_{0}+1
$$

Step 2. Fix $x_{0}, r \leq\left|x_{0}\right| \leq s$, and let $\chi_{0}, \rho_{0, j}, u_{0}$ be as in Step 1. We shall construct now, around the graph of the trajectory $\gamma_{0}(\cdot) \doteq x\left(\cdot ; x_{0}, u_{0}\right)$, an open "increasing tube" starting from $\stackrel{\circ}{B}\left(x_{0}, \rho_{0}\right)$ which is positively 


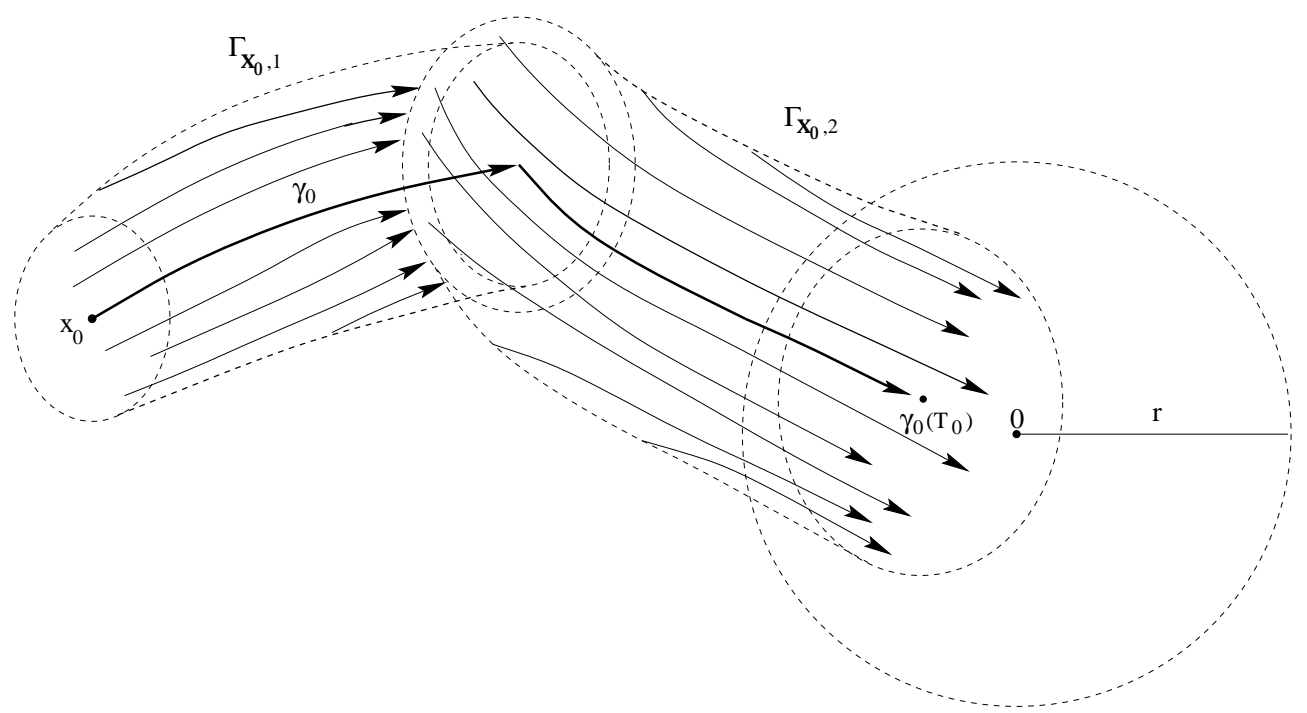

FiguRE 2

invariant with respect to the perturbed system $(4.6)_{\chi_{0}}$, and then define an admissible multivalued perturbation of a patchy vector field on such a tube. For any $j=1, \ldots N_{0}$, and for any fixed $\bar{x} \in \mathbb{R}^{n}$, denote $\mathcal{A}_{j}(\bar{x}, t)$ the attainable set for

$$
\dot{x} \in f\left(x, k_{0, j}\right)+B\left(0,2 \chi_{0}\right)
$$

at time $t \geq 0$ i.e., the set of all points $x=\gamma(t)$ where $\gamma$ is any Carathéodory trajectory of (4.10) defined on some interval $[0, \tau), t<\tau$, with $\gamma(0)=\bar{x}$. Define the sets

$$
\begin{aligned}
& \Gamma_{x_{0}, j} \doteq \quad \bigcup \quad \mathcal{A}_{j}(\bar{x}, t), \quad 1 \leq j<N_{0} \\
& \begin{array}{c}
\bar{x} \in \stackrel{\circ}{B}\left(\gamma_{0}\left(t_{0, j-1}\right), \rho_{0, j}\right) \\
0 \leq t \leq t_{0, j}-t_{0, j-1}
\end{array} \\
& \Gamma_{x_{0}, N_{0}} \doteq \underset{\substack{\bar{x} \in B\left(\gamma_{0}\left(t_{0, N_{0}-1}\right), \rho_{0, N_{0}}\right) \\
0 \leq t \leq T_{0}+\rho_{0}-t_{0, N_{0}-1}}}{\bigcup} \mathcal{A}_{N_{0}}(\bar{x}, t), \\
& \Delta_{x_{0}} \doteq \bigcup_{j=1}^{N_{0}} \Gamma_{x_{0}, j}
\end{aligned}
$$

Observe that, by the regularity of $f$, for any $\bar{z} \in \partial \Gamma_{x_{0}, j} \backslash \Gamma_{x_{0}, j+1}$ one can find some cone

$$
C_{\bar{z}}=\left\{y \in \mathbb{R}^{n}: \exists 0 \leq \lambda<\bar{\lambda} \quad \text { s.t } \quad|y-\lambda f(\bar{z})| \leq \lambda \chi_{0}\right\}, \quad \bar{\lambda}>0,
$$

such that

But, since

$$
C_{\bar{z}} \backslash\{\bar{z}\} \subset\left(\bigcup_{t \geq 0} \stackrel{\circ}{\mathcal{A}}_{j}(\bar{z}, t)\right) \backslash \Gamma_{x_{0}, j+1}
$$

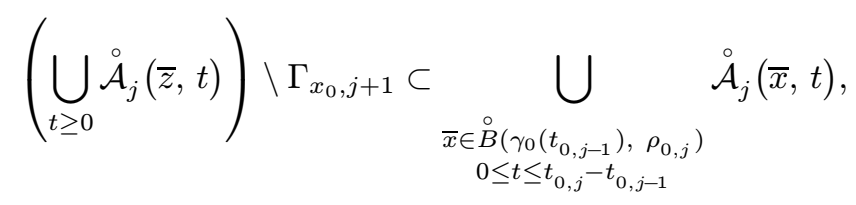


it follows that

which implies

$$
C_{\bar{z}} \backslash\{\bar{z}\} \subset \Gamma_{x_{0}, j}
$$

$$
\left\langle f\left(\bar{z}, k_{0, j}\right)+v, \mathbf{n}(\bar{z})\right\rangle<0 \quad \forall v \in B\left(0, \chi_{0}\right), \quad \bar{z} \in \partial \Gamma_{x_{0}, j} \backslash \Gamma_{x_{0}, j+1}, \quad 1 \leq j<N_{0}
$$

(denoting with $\mathbf{n}(\bar{z})$ the outer normal to $\left.\Gamma_{x_{0}, j}\right)$. With similar arguments one can verify that

$$
\left\langle f\left(\bar{z}, k_{0, N_{0}}\right)+v, \mathbf{n}(\bar{z})\right\rangle<0 \quad \forall v \in B\left(0, \chi_{0}\right),
$$

Now, let $h$ be a smooth vector field such that

$$
h(x)=-\mathbf{n}(x) \quad \forall x \in \partial \Gamma_{x_{0}, N_{0}},
$$

then construct a smooth scalar function $\phi: \Delta_{x_{0}} \mapsto[0,1]$ such that

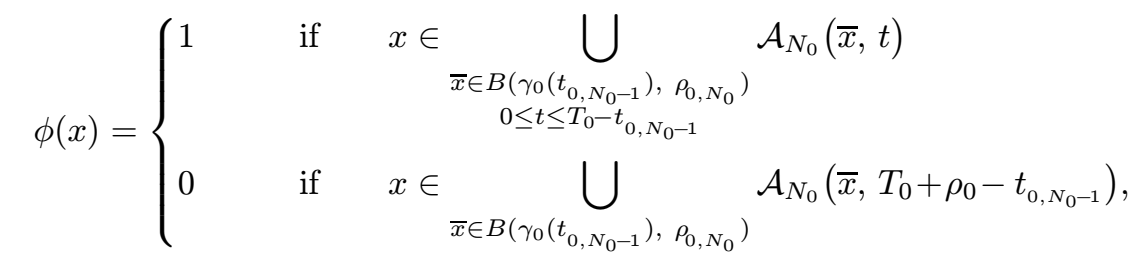

and define the interpolated field

$$
g_{0, N_{0}} \doteq \phi(x) f\left(x, k_{0, N_{0}}\right)+(1-\phi(x)) h(x)
$$

Finally, denote $g_{0, j}=g_{x_{0}, j}$ the vector field on $\mathbb{R}^{n}$ defined by

$$
g_{0, j}(x) \doteq f\left(x, k_{0, j}\right) \quad 1 \leq j<N_{0}
$$

and $g_{0}=g_{x_{0}}$ the vector field on $\Delta_{x_{0}}$ defined by

$$
g_{0}(x) \doteq g_{0, j}(x) \quad \text { if } \quad x \in \Gamma_{x_{0}, j} \backslash \bigcup_{\ell>j} \Gamma_{x_{0}, \ell}
$$

Then, using (4.13-4.14) and in view of Remarks 2.1 and 2.2, we deduce that the following holds.

i) The triple $\left(\Delta_{x_{0}}, g_{0},\left(\Gamma_{x_{0}, j}, g_{0, j}\right)_{1 \leq j \leq N_{0}}\right)$ is a smooth patchy vector field on $\Delta_{x_{0}}$.

ii) The multivaled map $G_{0}: \Delta_{x_{0}} \mapsto 2^{\mathbb{R}^{n}}$ defined by

$$
G_{0}(x) \doteq g_{0}(x)+B\left(0, \chi_{0}\right) \quad x \in \Delta_{x_{0}},
$$

is an admissible multivalued perturbation of $\left(\Delta_{x_{0}}, g_{0},\left(\Gamma_{x_{0}, j}, g_{0, j}\right)_{1 \leq j \leq N_{0}}\right)$ according with Definition 2.4 . 
iii) By Proposition 3.2-i), for any Carathéodory trajectory $\gamma$ of

$$
\dot{x} \in G_{0}(x)
$$

starting from some point $\bar{x} \in \Gamma_{x_{0}, j}$ at time $t=0$, there exists an $H$-tuple of points $\tilde{t}_{1}=0<\cdots<\tilde{t}_{H}=$ $T_{0}-t_{0, j-1}\left(H \leq N_{0}-j\right)$, and indices $j \leq \ell_{1}<\cdots<\ell_{H} \leq N_{0}$, such that

$$
\gamma(t) \in \Gamma_{x_{0}, \ell_{h}} \backslash \bigcup_{k>\ell_{h}} \Gamma_{x_{0}, k} \quad \forall t \in\left(\tilde{t}_{h-1}, \tilde{t}_{h}\right] .
$$

Moreover, because of $(4.3,4.5,4.8,4.9)$, one has

$$
\begin{aligned}
|\gamma(t)| & \leq\left|\gamma(t)-\gamma_{0}\left(t+t_{0, j-1}\right)\right|+\left|\gamma_{0}\left(t+t_{0, j-1}\right)\right| \quad \forall t \in\left[0, T_{0}-t_{0, j-1}\right] \\
& <\rho_{0, N_{0}+1}+M_{0}
\end{aligned}
$$

and

$$
\begin{aligned}
\left|\gamma\left(T_{0}-t_{0, j-1}\right)\right| & \leq\left|\gamma\left(T_{0}-t_{0, j-1}\right)-\gamma_{0}\left(T_{0}\right)\right|+\left|\gamma_{0}\left(T_{0}\right)\right| \\
& <\rho_{0, N_{0}+1}+r / 2<r .
\end{aligned}
$$

Step 3. For any fixed $x_{0}, r \leq\left|x_{0}\right| \leq s$, let $T_{x_{0}}, M_{x_{0}}, \chi_{x_{0}}, \rho_{x_{0}, j}, k_{x_{0}, j}$, be as in Step 1. The family of open tubes $\left\{\Delta_{x_{0}}: r \leq\left|x_{0}\right| \leq s\right\}$ constructed in Step 2 forms an open covering of the compact set $B(0, s) \backslash \stackrel{\circ}{B}(0, r)$. Let

$$
\left\{\Delta_{i}=\Delta_{x_{i}}: i=1, \ldots, N(r, s)\right\}, \quad \Delta_{i}=\bigcup_{j=1}^{N_{i}} \Gamma_{i, j}, \quad \Gamma_{i, j}=\Gamma_{x_{i}, j},
$$

be a finite subcover. Denote

$$
g_{i, j}(x) \doteq f\left(x, k_{i, j}\right), \quad k_{i, j}=k_{x_{i}, j} .
$$

The index set

$$
\mathcal{A}=\left\{(i, j): i=1, \ldots, N(r, s), \quad j=0, \ldots, N_{i}\right\}
$$

can be totally ordered by letting

$$
(i, j) \prec(h, k) \quad \text { if either } \quad i<h \quad \text { or else } \quad i=h, \quad j<k \text {. }
$$

Then, if we set

$$
D^{r, s} \doteq \bigcup_{i=1}^{N(r, s)} \Delta_{i}
$$

and define the map $U^{r, s}: D^{r, s} \mapsto K$ by

$$
U^{r, s}(x)=k_{i, j} \quad \text { if } \quad x \in \Gamma_{i, j} \backslash \bigcup_{(h, k)>(i, j)} \Gamma_{h, k},
$$

thanks to the properties established in Step 2 and in view of Remarks 2.1 and 2.3, the triple $\left(D^{r, s}, U^{r, s}\right.$, $\left.\left(\Gamma_{i, j}, k_{i, j}\right)_{(i, j) \in \mathcal{A}}\right)$ is a patchy feedback control on $D^{r, s}$. Moreover, if we let $g^{r, s}$ denote the corresponding patchy vector field defined by

the multivalued map $G^{r, s}: D^{r, s} \mapsto 2^{\mathbb{R}^{n}}$ defined by

$$
g^{r, s}(x)=f\left(x, U^{r, s}(x)\right),
$$

$$
G^{r, s}(x) \doteq g^{r, s}(x)+B\left(0, \chi^{r, s}\right), \quad \chi^{r, s}=\min _{1 \leq i \leq N(r, s)} \chi_{x_{i}}
$$




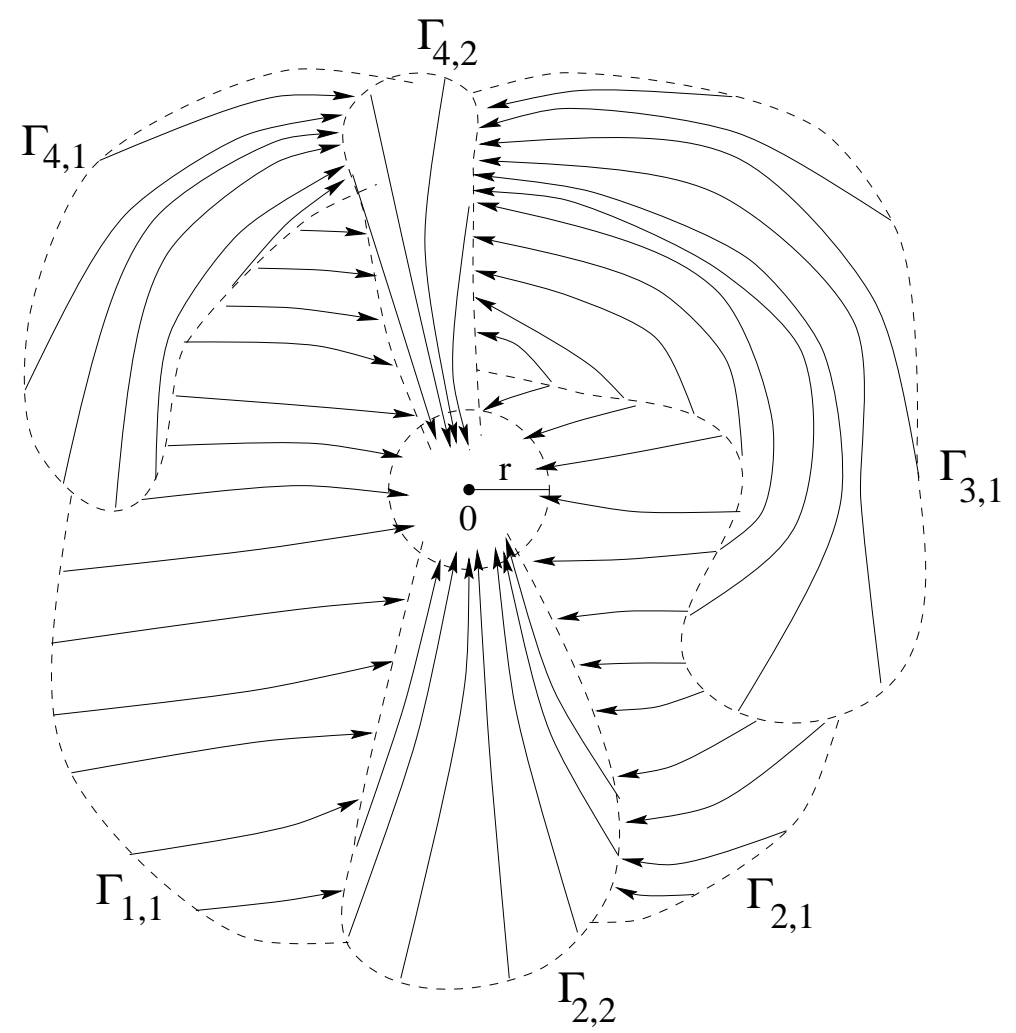

FiguRE 3

is an admissible perturbation of $\left(D^{r, s}, g^{r, s},\left(\Gamma_{i, j}, g_{i, j}\right)_{(i, j) \in \mathcal{A}}\right)$ according with Definition 2.4 and Remark 2.2. Thus, if we set

$$
\begin{aligned}
T(r, s) & \doteq \sum_{i=1}^{N(r, s)} T_{x_{i}}, \\
R(r, s) & \doteq \sup _{1 \leq i \leq N(r, s)}\left[\rho_{x_{i}, N_{i}+1}+M_{x_{i}}\right],
\end{aligned}
$$

by Proposition 3.2-i) and thanks to the properties established in Step 2 we deduce that, for any Carathéodory trajectory $\gamma$ of

$$
\dot{x} \in G^{r, s}(x)
$$

starting from some point $\bar{x} \in \in D^{r, s} \backslash \stackrel{\circ}{B}(0, r)$ at time $t=0$, there exists an $H$-tuple of points $\tilde{t}_{1}=0<\cdots<$ $\tilde{t}_{H} \leq T(r, s) \quad(H \leq N(r, s)+1)$, and indices $1 \leq \ell_{1}<\cdots<\ell_{H} \leq N(r, s)$, such that there holds

$$
\begin{gathered}
\tilde{t}_{h}-\tilde{t}_{h-1}<T_{x_{\ell_{h}}}, \\
\gamma(t) \in \Delta_{\ell_{h}} \backslash \bigcup_{k>\ell_{h}} \Delta_{k} \\
|\gamma(t)|<\rho_{x_{\ell_{h}}, N_{\ell_{h}}+1}+M_{x_{\ell_{h}}} \\
\gamma\left(\tilde{t}_{H}\right)<r .
\end{gathered}
$$

In particular, from (4.22) it follows

$$
|\gamma(t)|<R(r, s) \quad \forall t \in\left[0, \tilde{t}_{H}\right]
$$


which, together with $(4.23)$, yields $(4.1,4.2)$ concluding the proof.

Proposition 4.2. Let system (1.1) be globally asymptotically controllable to the origin. Then, for any fixed $\varepsilon>0$ there exists $\delta=\delta(\varepsilon)>0$ such that for any $0<r<s \leq \delta$ one can find $T=T(r, s)>0, R=R(r, s)>$ $0, \chi=\chi(r, s)>0$, and a patchy feedback control $U=U^{r, s}: D^{r, s} \mapsto K$ as in Proposition 4.1, with

$$
R(r, s)<\varepsilon
$$

Proof. We shall implement the same construction of the proof of Proposition 4.1 the only difference consisting in the more careful choice of the stabilizing open-loop control $u_{x_{0}}=u_{0}$ associated to each point $x_{0}, r \leq\left|x_{0}\right| \leq s$. Fix $\varepsilon>0$. Since (1.1) is globally asymptotically controllable, there will be some constant

$$
0<\delta=\delta(\varepsilon)<\varepsilon
$$

such that, for any fixed $0<r<s \leq \delta$ and for each $x_{0},\left|x_{0}\right| \leq s$, we can find a piecewise constant admissible control $u_{0}=u_{x_{0}}$ and some constant $T_{0}=T_{x_{0}}>0$ such that there holds (4.3) together with

$$
\left|\gamma_{0}(t)\right|<\varepsilon / 2 \quad \forall t \in\left[0, T_{0}\right]
$$

This means that the constant $M_{0}=M_{x_{0}}$ defined in (4.5) satisfies the uniform bound

$$
M_{0}<\varepsilon / 2
$$

But then, performing the same construction developed in the previous proposition and adopting the same notation, since by (4.9) one has

$$
\rho_{x_{0}, N_{0}+1}<r / 2 \leq \delta / 2
$$

from $(4.22,4.26,4.27)$ we deduce that, for any fixed $\bar{x}, r \leq|\bar{x}| \leq s$, and for any Carathéodory trajectory $\gamma$ of (4.20) starting from $\bar{x}$, there exists $0<t_{\gamma}<T(r, s)$, such that

$$
\begin{aligned}
\left|\gamma\left(t_{\gamma}\right)\right| & <r \\
|\gamma(t)| & <\delta / 2+\varepsilon / 2<\varepsilon \quad \forall 0 \leq t \leq t_{\gamma},
\end{aligned}
$$

proving (4.25).

Theorem 1. If the system (1.1) is asymptotically controllable, then it admits an asymptotically stabilizing, piecewise constant patchy-feedback, that is robust with respect to external disturbances.

Proof. Let $\left(\delta_{n}\right)_{n \in \mathbb{N}}$ be a decreasing sequence of positive number chosen according with Proposition 4.2 so that, for any fixed $0<r<s \leq \delta_{n}$, one can find $T=T(r, s)>0, R=R(r, s)>0, \chi=\chi(r, s)>0$, and a patchy feedback control $U=U^{r, s}: D^{r, s} \mapsto K$ as in Proposition 4.1, with

$$
R(r, s)<1 / n
$$

Define inductively two decreasing sequences of positive numbers $\left(s_{n}\right)_{n \in \mathbb{N}},\left(r_{n}\right)_{n \in \mathbb{N}}$, converging to zero and satisfying

$$
r_{n-1}<s_{n} \leq \delta_{n}
$$

and two increasing sequences of positive number $\left(s_{-n}\right)_{n \in \mathbb{N}},\left(r_{-n}\right)_{n \in \mathbb{N}}$, diverging to infinity and satisfying

$$
r_{-(n+1)}<s_{-n}
$$


For any $n \in \mathbb{Z}$, let $T_{n}=T\left(r_{n}, s_{n}\right)>0, R_{n}=R\left(r_{n}, s_{n}\right)>0, \chi_{n}=\chi\left(r_{n}, s_{n}\right)>0$, be defined as in Proposition 4.1 in connection with a patchy feedback control

$$
\left(D^{r_{n}, s_{n}}, U^{r_{n}, s_{n}},\left(\Gamma_{i}^{n}, k_{i}^{n}\right)_{1 \leq i \leq N_{n}}\right),
$$

and satisfying

$$
R\left(r_{n}, s_{n}\right)<1 / n \quad \forall n \in \mathbb{N} .
$$

The index set

$$
\mathcal{B}=\left\{(n, i): n \in \mathbb{Z}, \quad i=1, \ldots, N_{n},\right\}
$$

can be totally ordered by letting

$$
(n, i) \prec(m, j) \quad \text { if either } \quad n<m \quad \text { or else } \quad n=m, \quad i<j .
$$

Then, if we define the map $U: R^{n} \backslash\{0\} \mapsto K$ by setting

$$
U(x)=k_{i}^{n} \quad \text { if } \quad x \in \Gamma_{i}^{n} \backslash \bigcup_{(m, j)>(n, i)} \Gamma_{j}^{m},
$$

thanks to the properties established in Proposition 4.1 and in view of Remarks 2.1, 2.3, the triple

$$
\left(R^{n} \backslash\{0\}, U,\left(\Gamma_{i}^{n}, k_{i}^{n}\right)_{(i, n) \in \mathcal{B}}\right)
$$

is a patchy feedback control on $\mathbb{R}^{n} \backslash\{0\}$. Next, set

$$
\tilde{\chi}(x)=\chi_{n} \quad \text { if } \quad x \in D^{r_{n}, s_{n}} \backslash \bigcup_{m>n} D^{r_{m}, s_{m}},
$$

and consider the inf-convolution of $\tilde{\chi}$ with $|\cdot|$, i.e. the map $\chi: \mathbb{R}^{n} \backslash\{0\} \rightarrow R^{+}$defined by

$$
\chi(x)=\inf _{y \in \mathbb{R}^{n} \backslash\{0\}}\{\tilde{\chi}(y)+|y-x|\} .
$$

One can easily verify that $\chi$ is Lipschitzian with constant 1 and clearly it satisfies

$$
\chi(x) \leq \tilde{\chi}(x) \quad \forall x \in \mathbb{R}^{n} \backslash\{0\} .
$$

Therefore, if we let $g(x)=f(x, U(x))$ denote the patchy vector field associated with (4.31), and set

$$
g_{i}^{n}(x)=f\left(x, k_{i}^{n}\right) \quad \text { if } \quad x \in \Gamma_{i}^{n} \backslash \bigcup_{(m, j)>(n, i)} \Gamma_{j}^{m},
$$

the multivalued map $G: R^{n} \backslash\{0\} \mapsto 2^{\mathbb{R}^{n}}$ defined by

$$
G(x) \doteq g(x)+B(0, \chi(x))
$$

is an admissible perturbation of $\left(R^{n} \backslash\{0\}, g,\left(\Gamma_{i}^{n}, g_{i}^{n}\right)_{(i, n) \in \mathcal{B}}\right)$ according with Definition 2.4 and Remark 2.2.

Now, given $x_{0} \in \mathbb{R}^{n} \backslash\{0\}$, consider any Carathéodory trajectory $\gamma$ of (2.15), with $G$ as above, starting from $x_{0}$. Fix an arbitrary $0<r<\left|x_{0}\right|$ and let $m<n, 0<n$, be such that

$$
x_{0} \in D^{r_{m}, s_{m}} \backslash \bigcup_{p>m} D^{r_{p}, s_{p}}, \quad \quad r_{n}<r .
$$


Then, by Proposition 3.2)-i), we deduce that there exists an $H$-tuple of points $\tilde{t}_{1}=0<\cdots<\tilde{t}_{H} \leq \sum_{p=m}^{n} T_{p}\left(H \leq 1+\sum_{p=m}^{n} N_{p}\right)$, and induces $m \leq n_{h} \leq n, 1 \leq \ell_{h} \leq N_{n_{h}}$, such that there holds

$$
\begin{aligned}
& \tilde{t}_{h}-\tilde{t}_{h-1}<T_{n_{h}}, \\
& \gamma(t) \in \Gamma_{\ell_{h}}^{n_{h}} \backslash \bigcup_{(p, k)>\left(n_{h}, \ell_{h}\right)} \Gamma_{p}^{k} \quad \forall t \in\left(\tilde{t}_{h-1}, \tilde{t}_{h}\right], \\
& |\gamma(t)| \leq R\left(r_{n_{h}}, s_{n_{h}}\right) \\
& \gamma\left(\tilde{t}_{H}\right)<r .
\end{aligned}
$$

In particular, (4.34) yields (2.13) being $r$ arbitrary, while from (4.33) and (4.30) one can recover the Lyapunov stability, concluding the proof.

Remark 4.1. The idea of using a piecewise-constant feedback law to stabilize an asymptotycally controllable system has been recently employed also by Nikitin in [21]. The feedback synthesis $U=U(x)$ outlined in [21] stabilizes the system (1.1), over a given compact subset $\mathcal{K}$ of the state space $\mathbb{R}^{n}$, in the following sense. For every initial data $x_{0} \in \mathcal{K}$, there exists at least one (Carathéodory) solution to the Cauchy problem

$$
\dot{x}=f(x, U(x)), \quad x(0)=x_{0},
$$

which asymptotycally converges to the equilibrium state $x^{*}$. However, since the resulting vector field $f(x, U(x))$, in general, does not satisfy any transversality condition, one can produce examples where the algorithm proposed by Nikitin generates a feedback control with the following property:

for every initial data $x_{0}$ in the starting domain $\mathcal{K}$, the Cauchy problem (4.35) has infinitely many Carathéodory solutions. Some of these solutions asymptotically approache $x^{*}$, others become eventually periodic and have no limit as $t \rightarrow \infty$.

Notice that, even if one introduces an appropriate definition of solution so to rule out those trajectories which do not approach the equilibrium state, a feedback of this type will be by no means robust w.r.t. dynamic perturbations. This behaviour is illustrated by the following

Example 4.1. Consider a two-dimensional system with scalar controls

$$
\dot{x}=f(x, u), \quad x=\left(x_{1}, x_{2}\right) \in \mathbb{R}^{2}, \quad u \in K \subset \mathbb{R},
$$

and assume that there exist control values $k_{1}, k_{2} \in K$ such that

$$
\begin{aligned}
& f\left(x, k_{1}\right)=\left(x_{2}-2,4-x_{1}\right), \\
& f\left(x, k_{2}\right)=(-1,0) .
\end{aligned}
$$

We are interested in a problem of semiglobal practical stabilization for the system (4.36) over the domain

$$
\mathcal{K}=\left\{\left(x_{1}, x_{2}\right): \quad 2 \leq x_{1} \leq 3, \quad x_{2}=2\right\}
$$

Namely, we look for a feedback $U=U(x)$ which steers any point $x_{0}$ of $\mathcal{K}$ into the unit ball $B_{1} \doteq B(0,1)$, within finite time. Following the construction in [21] we define a feedback law $U^{*}=U^{*}(x)$ by setting

$$
U^{*}\left(x_{1}, x_{2}\right)=\left\{\begin{array}{lll}
k_{1} & \text { if } & 1<\left|x_{2}\right|, \\
k_{2} & \text { if } & \left|x_{2}\right| \leq 1 .
\end{array}\right.
$$

One can easily check that any trajectory of the corresponding closed-loop system, starting at a point of $\mathcal{K} \backslash\{B\}, B=(3,2)$, first loops around $P=(4,2)$ untill it reaches a point of the strip $S=\left\{x:\left|x_{2}\right| \leq 1\right\}$, next follows the integral curve of $f\left(x, k_{2}\right)$ and thus reaches the ball $B_{1}$. On the other hand, the trajectory that 


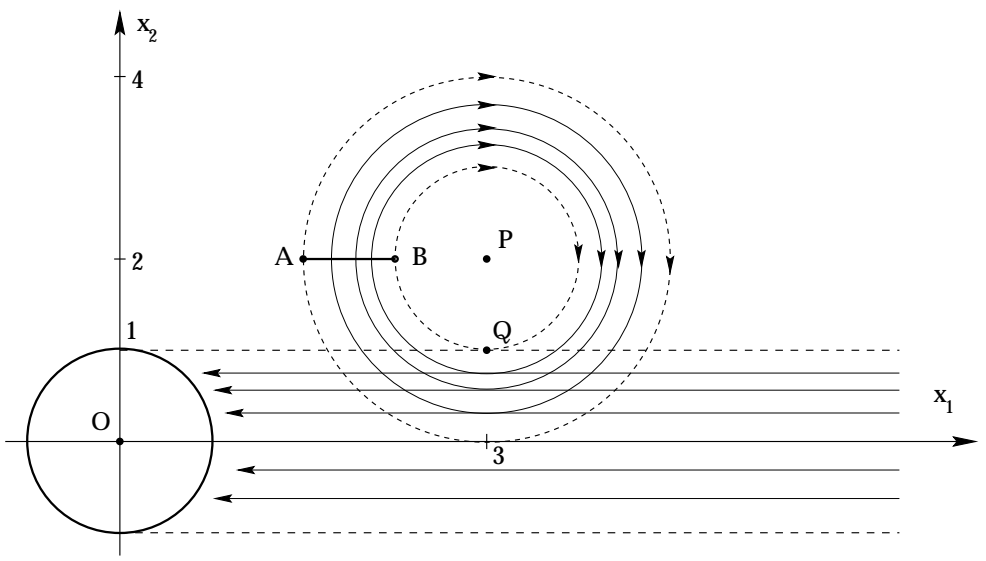

FiguRe 4

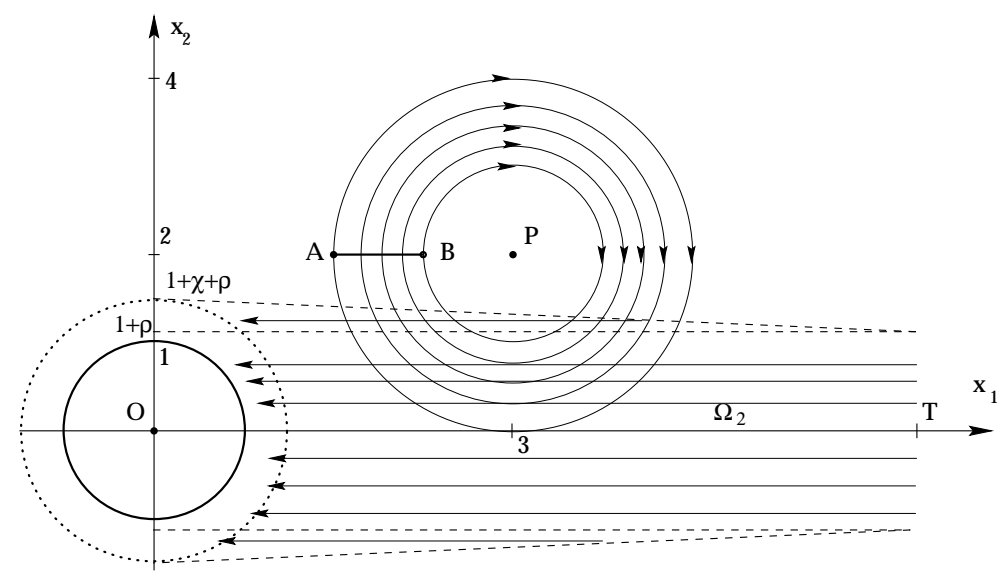

FiguRE 5

starts at $B$ crosses the strip $S$ at $Q=(4,1)$ where the two fields $f\left(x, k_{1}\right), f\left(x, k_{2}\right)$ coincide. Hence it can either keep following the field $f\left(x, k_{1}\right)$ untill it crosses again the strip $S$, or else can immediately enter the strip $S$ and thus follow the field $f\left(x, k_{2}\right)$. Therefore the closed loop system admits infinitely many trajectories starting at the point $(3,2)$. Some of these actually reach the ball $B_{1}$ in finite time, others keep spinning around the point $P$ forever.

Let's implement now our construction of a patchy-feedback for this system. We shall define a feedback that steers all the states in $\mathcal{K}$ into an arbitrary small neighborhood of $B_{1}$. Fix some $0<\rho \ll 1$ and then, for any $0<\chi \ll 1$, define a feedback law $U_{\chi}=U_{\chi}(x)$ by setting

$$
U_{\chi}\left(x_{1}, x_{2}\right)=\left\{\begin{array}{lll}
k_{1} & \text { if } & (1+\chi+\rho)-\frac{\chi}{T} \cdot x_{1} \leq\left|x_{2}\right|, \\
k_{2} \quad \text { if } & \left|x_{2}\right|<(1+\chi+\rho)-\frac{\chi}{T} \cdot x_{1},
\end{array}\right.
$$

where $T$ is some constant $>6$. The resulting field $g(x)=f\left(x, U_{\chi}(x)\right)$ is a patchy vector field associated to the pair of patches $\left(\Omega_{1}, g_{1}\right),\left(\Omega_{2}, g_{2}\right)$, with

$$
\begin{aligned}
\Omega_{1} & =\mathbb{R}^{2}, & \Omega_{2} & =\left\{\left(x_{1}, x_{2}\right):\left|x_{2}\right|<(1+\chi+\rho)-\frac{\chi}{T} \cdot x_{1}\right\} \\
g_{1}(x) & =f\left(x, k_{1}\right), & g_{2}(x) & =f\left(x, k_{2}\right) .
\end{aligned}
$$


In this case any trajectory $\gamma$ of

$$
\dot{x}=g(x)
$$

that starts at a point in $\mathcal{K}$, after looping around $P$ crosses transversally the boundary of $\Omega_{2}$. As a result, every Carathéodory solution of (4.37), starting at time $t=0$ from a point in $\mathcal{K}$, reaches the ball $B(0,1+(\chi+\rho))$, in finite time. Moreover, if we choose the constant $\chi$ sufficiently small, one can easily verify that also all the Carathéodory solutions of the differential inclusion

$$
\dot{x} \in g(x)+B(0, \chi / 2)
$$

starting from a point in $\mathcal{K}$, reach the ball $B(0,1+(\chi+\rho))$ in finite time. Thus, such a feedback is robust w.r.t. small external dynamic perturbations.

\section{REFERENCES}

[1] Z. Artstein, Stabilization with relaxed controls. Nonlinear Anal. 7 (1983) 1163-1173.

[2] A. Bacciotti, Local stabilizability of nonlinear control systems. Series on advances in mathematics for applied sciences $\mathbf{8}$, World Scientific, Singapore (1992).

[3] R.W. Brockett, Asymptotic stability and feedback stabilization, in Differential Geometric Control Theory, R.W. Brockett, R.S. Millman and H.J. Sussmann, Eds., Birkhauser, Boston (1983) 181-191.

[4] F.H. Clarke, Yu.S. Ledyaev, E.D. Sontag and A.I. Subbotin, Asymptotic controllability implies feedback stabilization. IEEE Trans. Automat. Control 42 (1997) 1394-1407.

[5] F.H. Clarke, Yu.S. Ledyaev, L. Rifford and R.J. Stern, Feedback stabilization and Lyapunov functions, to appear.

[6] F.H. Clarke, Yu.S. Ledyaev, R.J. Stern and P.R. Wolenski, Qualitative properties of trajectories of control systems: A survey. J. Dynamic Control Systems 1 (1995) 1-47.

[7] F.H. Clarke, Yu.S. Ledyaev, R.J. Stern and P.R. Wolenski, Nonsmooth analysis and control theory 178, Springer-Verlag, New York (1998).

[8] G. Colombo, On extremal solutions of differential inclusions. Bull. Polish. Acad. Sci. 40 (1992) 97-109.

[9] J.-M. Coron, A necessary condition for feedback stabilization. Systems Control Lett. 14 (1990) 227-232.

[10] J.-M. Coron and L. Rosier, A relation between continuous time-varying and discontinuous feedback stabilization. J. Math. Systems, Estimation, and Control 4 (1994) 67-84.

[11] J.-M. Coron, Global asymptotic stabilization for controllable systems without drift. Math. of Control, Signals, and Systems 5 (1992) 295-312.

[12] J.-M. Coron, Stabilization in finite time of locally controllable systems by means of continuous time-varying feedback laws. SIAM J. Control Optim. 33 (1995) 804-833.

[13] J.-M. Coron, L. Praly and A. Teel, Feedback stabilization of nonlinear systems: sufficient conditions and Lyapunov and input-output techniques, in Trends in Control: A European Perspective, A. Isidori, Eds., Springer, London (1995) $293-348$.

[14] A.F. Filippov, Differential Equations with Discontinuous Right-Hand Sides, Kluwer Acad. Publ. (1988).

[15] O. Hájek, Discontinuos differential equations, I-II. J. Differential Equations 32 (1979) 149-185.

[16] H. Hermes, Discontinuous vector fields and feedback control, in Differential Equations and Dynamical Systems, J.K. Hale and J.P. La Salle, Eds., Academic Press, New York, (1967) 155-165.

[17] H. Hermes, On the synthesis of stabilizing feedback controls via Lie algebraic methods. SIAM J. Control Optim. 10 (1980) $352-361$.

[18] N.N. Krasovskii and A.I. Subbotin, Positional differential games, Nauka, Moscow, (1974) [in Russian]. Revised English translation: Game-theoretical control problems, Springer-Verlag, New York (1988).

[19] Yu.S. Ledyaev and E.D. Sontag, A remark on robust stabilization of general asymptotically controllable systems, in Proc. Conf. on Information Sciences and Systems (CISS 97), Johns Hopkins, Baltimore, MD (1997) 246-251.

[20] Yu.S. Ledyaev and E.D. Sontag, A Lyapunov characterization of robust stabilization. J. Nonlinear Anal. to appear.

[21] S. Nikitin, Piecewise-constant stabilization. SIAM J. Control Optim. to appear.

[22] E.P. Ryan, On Brockett's condition for smooth stabilizability and its necessity in a context of nonsmooth feedback. SIAM $J$. Control Optim. 32 (1994) 1597-1604.

[23] E.D. Sontag and H.J. Sussmann, Remarks on continuous feedback, in Proc. IEEE Conf. Decision and Control, Aulbuquerque, IEEE Publications, Piscataway (1980) 916-921. 
[24] E.D. Sontag, Nonlinear regulation: The piecewise linear approach. IEEE Trans. Automat. Control 26 (1981) $346-358$.

[25] E.D. Sontag, Feedback stabilization of nonlinear systems, in Robust Control of Linear Systems and Nonlinear Control, M.A. Kaashoek, J.H. van Shuppen and A.C.M. Ran, Eds., Birkhäuser, Cambridge, MA (1990) 61-81.

[26] E.D. Sontag, Mathematical control theory, deterministic finite dimensional systems, Springer-Verlag, New York (1990).

[27] E.D. Sontag, Stability and stabilization: Discontinuities and the effect of disturbances, in Proc. NATO Advanced Study Institute - Nonlinear Analysis, Differential Equations, and Control (Montreal, Jul/Aug 1998), F.H. Clarke and R.J. Stern, Eds., Kluwer (1999) 551-598.

[28] H.J. Sussmann, Subanalytic sets and feedback control. J. Differential Equations 31 (1979) 31-52. 